TRANSACTIONS OF THE

AMERICAN MATHEMATICAL SOCIETY

Volume 359, Number 7, July 2007, Pages 3209-3237

S 0002-9947(07)04251-

Article electronically published on January 19, 2007

\title{
EQUILIBRIUM POINTS OF LOGARITHMIC POTENTIALS INDUCED BY POSITIVE CHARGE DISTRIBUTIONS. I. GENERALIZED DE BRUIJN-SPRINGER RELATIONS
}

\author{
JULIUS BORCEA
}

\begin{abstract}
A notion of weighted multivariate majorization is defined as a preorder on sequences of vectors in Euclidean space induced by the Choquet ordering for atomic probability measures. We characterize this preorder both in terms of stochastic matrices and convex functions and use it to describe the distribution of equilibrium points of logarithmic potentials generated by discrete planar charge configurations. In the case of $n$ positive charges we prove that the equilibrium points satisfy $\left(\begin{array}{l}n \\ 2\end{array}\right)$ weighted majorization relations and are uniquely determined by $n-1$ such relations. It is further shown that the Hausdorff geometry of the equilibrium points and the charged particles is controlled by the weighted standard deviation of the latter. By using finiterank perturbations of compact normal Hilbert space operators we establish similar relations for infinite charge distributions. We also discuss a hierarchy of weighted de Bruijn-Springer relations and inertia laws, the existence of zeros of Borel series with positive $l^{1}$-coefficients, and an operator version of the Clunie-Eremenko-Rossi conjecture.
\end{abstract}

\section{INTRODUCTION}

Let $\left\{z_{i}\right\}_{i \in \mathcal{N}}$ be a sequence of distinct points in a domain $\Omega \subseteq \mathbb{C}$, where $\mathcal{N} \subseteq \mathbb{N}$ with $|\mathcal{N}| \geq 2$. If at each $z_{i}$ we place a particle with charge $a_{i}$-actually, an infinitely long thin rod perpendicular to the plane with uniform charge distribution - then up to complex conjugation and a constant factor the resulting electrostatic force is given by

$$
f(z)=\sum_{i \in \mathcal{N}} \frac{a_{i}}{z-z_{i}}
$$

and the logarithmic potential generated by these charged particles is

$$
U(z)=\sum_{i \in \mathcal{N}} a_{i} \log \left|1-\frac{z}{z_{i}}\right|
$$

(cf. [23, p. 10]). If $|\mathcal{N}|=\infty$, then the series in (1.1) and (1.2) converge absolutely for all $z \in \Omega \backslash\left\{z_{i}\right\}_{i \in \mathcal{N}}$ provided that certain growth conditions are satisfied (see $\$ 3.3$ below). In this case $f$ is meromorphic while $U$ is subharmonic in $\Omega$. Since $\nabla U(z)=\overline{f(z)}$ the critical points of the potential coincide with the equilibrium

Received by the editors April 29, 2005.

2000 Mathematics Subject Classification. Primary 31A15; Secondary 30C15, 47A55, 60E15.

Key words and phrases. Logarithmic potentials, electrostatic equilibrium, Hausdorff geometry, multivariate majorization, compressions of normal operators.

(C)2007 American Mathematical Society Reverts to public domain 28 years from publication 
points of the field, that is, points upon which a free electron (or rod) once placed would remain. An important problem that goes back to Gauss is to describe the distribution and the relative geometry of the equilibrium points and charged particles. One of the main purposes of this paper is to study this problem by means of the Hausdorff distance and the notion of weighted multivariate majorization that we define in 92 as an extension of classical and matrix majorization. It is interesting to note that although our results are purely analytic or geometric in nature, they are obtained through a combination of the multilinear algebra and operator theoretic methods developed in [25, 26] and [25, 26, 28, respectively. The fact that such geometric properties could be established in this way was actually first noticed in loc. cit., where long-standing conjectures on the geometry of polynomials were solved by applying this type of technique. Below we make extensive use of the key observation made in the aforementioned papers, namely that studying zeros and critical points of complex polynomials - or, more generally, poles and zeros of logarithmic potentials generated by finite positive charge configurations - is equivalent to studying spectra of normal matrices and their compressions.

The weighted multivariate generalization of vector and matrix majorization introduced in $\$ 2$ is a natural preorder on finite sequences of vectors in Euclidean space induced by the Choquet ordering for nonnegative Radon measures (Definition 1). We characterize this new notion in terms of stochastic matrices and convex functions, and we recover in this way several related results on classical and multivariate majorization. In the process we use a Hahn-Banach type argument to give a new simple proof of a fundamental theorem of Sherman (Theorem 11).

In $₫ 3$ we state most of our main results. These describe the distribution and the relative location of equilibrium points of logarithmic potentials for discrete bounded planar configurations of positive charges and are of three different types. First, we consider $n$ positive charges and prove that the equilibrium points satisfy $\left(\begin{array}{l}n \\ 2\end{array}\right)$ weighted multivariate majorization relations and are in fact uniquely determined by $n-1$ such relations (Theorems 2 3). The higher de Bruijn-Springer relations thus obtained generalize some of the results of [25, 26 to arbitrary (not necessarily equal) positive charges. Second, we show that the relative Hausdorff geometry of the extended set of equilibrium points and the set of charged particles is controlled by the weighted standard deviation of the latter (Theorems 45). For equal charges the bounds given by these theorems are much better and also more natural than those predicted by the famous Sendov conjecture for complex polynomials and its converse; see, e.g., 3. As we explain in the sequel [4] to this paper, these results also seem to suggest that the Hausdorff geometry is governed by natural dispersion measures associated with the given charge configuration. Finally, we establish weighted multivariate majorization relations for essentially any bounded discrete set of positively charged particles with finite total charge for which equilibrium points are known to exist (Theorems 66 17). We further conjecture that equilibrium points actually always exist under some mild assumptions (Conjectures 23 in $\sqrt[66]{6}$ ).

The proofs of the aforementioned results are given in $\$ 4$ As we already pointed out, for finite charge configurations these are based on the multilinear algebra and matrix theory techniques of $[25,26,28]$. In the process we are led to consider several natural related questions, such as a hierarchy of inertia laws for finite systems of planar charged particles that we propose and discuss in 95 (Conjecture 11). This would yield a whole new series of weighted generalized de Bruijn-Springer relations. 
The techniques mentioned above actually extend to the case of infinite discrete distributions of charge, which allows us to make use of operator theoretic methods such as compressions of compact normal operators on separable Hilbert space in order to deal with this case. In this way we arrive at a great many related questions on the interplay between value distribution of meromorphic functions and variations of discrete spectra of compact normal Hilbert space operators under finite-rank perturbations. In $\$ 67$ we give an operator theoretic approach to the well-known Clunie-Eremenko-Rossi conjecture [8] dealing with the existence of zeros of certain Borel series and we consider possible extensions of this conjecture as well as their potential implications (Conjectures 46).

\section{WEIGHTED MULTIVARIATE MAJORIZATION}

The concept of majorization was first studied by economists early in the twentieth century as a means for altering the unevenness of distribution of wealth or income. The majorization preorder on $n$-tuples of real numbers - also known as the strong spectral order, vector majorization or classical majorization - was used by Hardy, Littlewood and Pólya in their study of analytic inequalities [19] and by Schur in his work on the spectra of positive semidefinite Hermitian operators (cf. [12]). Classical majorization has since become an important tool in analysis, combinatorics and statistics [27] and has recently found remarkable applications to quantum mechanics and entanglement transformations in quantum computation and information theory (see [2] and references therein). The strong spectral order essentially quantifies the intuitive notion that the components of a real $n$-vector $\mathbf{x}$ are "less spread out" than the components of another such vector $\mathbf{y}$. Several matrix versions of this notion have been proposed and studied in various contexts (cf., e.g., 9] and 27]).

In this section we generalize classical and matrix majorization by introducing a notion of weighted multivariate majorization that we shall use throughout both this paper and its sequel [4].

Notation 1. Let $k \in \mathbb{N}$. For each $n \in \mathbb{N}$ we define

$$
\begin{aligned}
& \mathcal{X}_{n}^{k}=\left\{\left(\mathbf{x}_{1}, \ldots, \mathbf{x}_{n}\right) \mid \mathbf{x}_{i} \in \mathbb{R}^{k}, 1 \leq i \leq n\right\}, \\
& \mathcal{A}_{n}=\left\{\left(a_{1}, \ldots, a_{n}\right) \mid a_{i} \in(0,1), 1 \leq i \leq n, \sum_{i=1}^{n} a_{i}=1\right\}, \\
& \mathbb{X}_{n}^{k}=\mathcal{X}_{n}^{k} \times \mathcal{A}_{n}
\end{aligned}
$$

and set

$$
\mathbb{X}^{k}=\bigcup_{n=1}^{\infty} \mathbb{X}_{n}^{k}
$$

Given $X=\left(\mathbf{x}_{1}, \ldots, \mathbf{x}_{m}\right) \in \mathcal{X}_{m}^{k}$ we denote by $X^{T}=\left(\mathbf{x}_{1}, \ldots, \mathbf{x}_{m}\right)^{T}$ its "transpose", i.e., the ordered $m$-tuple consisting of the vectors $\mathbf{x}_{1}, \ldots, \mathbf{x}_{m}$ written in column form. We identify $X^{T}$ with the $m \times k$ real matrix whose $i$-th row consists of the coordinates of the vector $\mathbf{x}_{i}$ in the standard basis of $\mathbb{R}^{k}$. Let finally $\mathbb{M}_{m, n}^{r s}$ be the set of all row stochastic $m \times n$ matrices.

The notion of weighted multivariate majorization defined below (see Definition 1) is motivated by the following theorem of Sherman [31. 
Theorem 1. Let $(X, \mathbf{a}) \in \mathbb{X}_{m}^{k}$ and $(Y, \mathbf{b}) \in \mathbb{X}_{n}^{k}$, where $X=\left(\mathbf{x}_{1}, \ldots, \mathbf{x}_{m}\right) \in \mathcal{X}_{m}^{k}$, $\mathbf{a}=\left(a_{1}, \ldots, a_{m}\right) \in \mathcal{A}_{m}, Y=\left(\mathbf{y}_{1}, \ldots, \mathbf{y}_{n}\right) \in \mathcal{X}_{n}^{k}$ and $\mathbf{b}=\left(b_{1}, \ldots, b_{n}\right) \in \mathcal{A}_{n}$. The following conditions are equivalent:

(i) For any (continuous) convex function $\Phi: \mathbb{R}^{k} \rightarrow \mathbb{R}$ one has

$$
\sum_{i=1}^{m} a_{i} \Phi\left(\mathbf{x}_{i}\right) \leq \sum_{j=1}^{n} b_{j} \Phi\left(\mathbf{y}_{j}\right) .
$$

(ii) There exists a matrix $R \in \mathbb{M}_{m, n}^{r s}$ such that

$$
\tilde{X}^{T}=R \tilde{Y}^{T} \text { and } \mathbf{b}=\mathbf{a} R,
$$

where $\tilde{X}^{T}$ is an $m \times k$ matrix and $\tilde{Y}^{T}$ is an $n \times k$ matrix obtained by some (and then any) ordering of the vectors in $X^{T}$ and $Y^{T}$, respectively.

The special case when $m=n$ and all weights are equal in Theorem 1 was long assumed to be an open question and appears only as an implicit conjecture in 27 , p. 433], which is the definite reference on majorization theory. Although essentially correct, Sherman's paper used rather complicated arguments and contained a series of misprints that required several subsequent errata, which may explain why the status of his proof seems to have remained unclear for such a long period. Below we present a short and simple proof of Theorem 1.

Before embarking on the actual proof let us note that condition (i) in Sherman's theorem may also be written in terms of the Choquet ordering for positive measures, a notion that we shall actually use in [4] (see Definition 9 in loc. cit.). Indeed, any pair $(X, \mathbf{a}) \in \mathbb{X}_{m}^{k}$ uniquely determines a discrete probability measure $\mu_{(X, \mathbf{a})}$ on $\mathbb{R}^{k}$ concentrating a mass $a_{i}$ at $\mathbf{x}_{i}, 1 \leq i \leq m$. Given two positive measures $\mu_{1}$ and $\mu_{2}$ on $\mathbb{R}^{k}$ one says that $\mu_{2}$ dominates $\mu_{1}$ in the Choquet ordering or that $\mu_{2}$ is a dilation of $\mu_{1}$, denoted $\mu_{1} \prec \mu_{2}$, if $\mu_{1}(\Phi) \leq \mu_{2}(\Phi)$ for any continuous convex function $\Phi$ on $\mathbb{R}^{k}$. Theorem 1 (i) may therefore be rewritten as $\mu_{(X, \mathbf{a})} \prec \mu_{(Y, \mathbf{b})}$. We refer to [5, 7, 13, 16, 29, for a detailed account of the Choquet ordering and various comparisons of nonnegative Radon measures and probability distributions in locally convex separable topological spaces. In particular, in the latter setting Sherman's theorem may in fact be viewed as a special case of the Cartier-Fell-Meyer theorem [7, 29, 5] and its subsequent extension due to Fischer-Holbrook [16. The most general result of this type, namely a basic representation theorem for convex domination of measures, seems to be contained in [13.

Proof of Theorem 1. Since the implication (ii) $\Rightarrow$ (i) is an immediate consequence of Jensen's inequality we shall only focus on the converse statement. Assume that condition (i) in Theorem 1 holds, let $X=\left(\mathbf{x}_{1}, \ldots, \mathbf{x}_{m}\right) \in \mathcal{X}_{m}^{k}, \mathbf{a}=\left(a_{1}, \ldots, a_{m}\right) \in$ $\mathcal{A}_{m}, Y=\left(\mathbf{y}_{1}, \ldots, \mathbf{y}_{n}\right) \in \mathcal{X}_{n}^{k}, \mathbf{b}=\left(b_{1}, \ldots, b_{n}\right) \in \mathcal{A}_{n}$, and define the following set:

$$
\mathcal{M}(X, Y)=\left\{A=\left(a_{i j}\right) \in \mathbb{M}_{m, n}^{r s} \mid \mathbf{x}_{i}=\sum_{j=1}^{n} a_{i j} \mathbf{y}_{j}, 1 \leq i \leq m\right\} .
$$

Lemma 1. $\mathcal{M}(X, Y)$ is a nonempty closed convex subset of $\mathbb{M}_{m, n}^{r s}$.

Proof. It is clear that $\mathcal{M}(X, Y)$ is closed and convex. To show that $\mathcal{M}(X, Y) \neq \emptyset$ it is enough to prove that if Theorem 1 (i) holds, then $\mathbf{x}_{i} \in \operatorname{conv}\left(\mathbf{y}_{1}, \ldots, \mathbf{y}_{n}\right)$ for $1 \leq i \leq m$, where $\operatorname{conv}\left(\mathbf{y}_{1}, \ldots, \mathbf{y}_{n}\right)$ is the convex hull of the vectors $\mathbf{y}_{1}, \ldots, \mathbf{y}_{n}$. Supposing that $\mathbf{x}_{l} \notin \operatorname{conv}\left(\mathbf{y}_{1}, \ldots, \mathbf{y}_{n}\right)$ for some $l \in\{1, \ldots, m\}$ let $d$ denote the 
Euclidean distance in $\mathbb{R}^{k}$ and consider the function $\Phi: \mathbb{R}^{k} \rightarrow \mathbb{R}$ given by $\Phi(\mathbf{u})=$ $d\left(\mathbf{u}, \operatorname{conv}\left(\mathbf{y}_{1}, \ldots, \mathbf{y}_{n}\right)\right)$. Note that $\Phi\left(\mathbf{x}_{l}\right)>0, \Phi\left(\mathbf{y}_{j}\right)=0$ for $1 \leq j \leq n$, and $\Phi$ is a convex function since $\operatorname{conv}\left(\mathbf{y}_{1}, \ldots, \mathbf{y}_{n}\right)$ is a nonempty convex subset of $\mathbb{R}^{k}$. It follows that $\sum_{i=1}^{m} a_{i} \Phi\left(\mathbf{x}_{i}\right)>\sum_{j=1}^{n} b_{j} \Phi\left(\mathbf{y}_{j}\right)$, which contradicts condition (i) of Theorem 1 Thus $\mathbf{x}_{i} \in \operatorname{conv}\left(\mathbf{y}_{1}, \ldots, \mathbf{y}_{n}\right)$ for $1 \leq i \leq m$, as required.

Given $M=\left(m_{i j}\right) \in \mathcal{M}(X, Y)$ we define a vector $\mathbf{s}(M)=\left(s_{1}(M), \ldots, s_{n}(M)\right) \in$ $\mathbb{R}^{n}$ by $s_{j}(M)=\sum_{i=1}^{m} a_{i} m_{i j}, 1 \leq j \leq n$. Set

$$
\mathcal{L}(X, Y)=\{\mathbf{s}(M) \mid M \in \mathcal{M}(X, Y)\} .
$$

Note that $\alpha \mathbf{s}(M)+(1-\alpha) \mathbf{s}(N)=\mathbf{s}(\alpha M+(1-\alpha) N)$ for any $M, N \in \mathcal{M}(X, Y)$ and $\alpha \in[0,1]$, so that $\mathcal{L}(X, Y)$ is a convex subset of $\mathbb{R}^{n}$. Moreover, since $\mathcal{M}(X, Y)$ is closed the same must be true for $\mathcal{L}(X, Y)$.

Lemma 2. With the above notations one has $\mathbf{b} \in \mathcal{L}(X, Y)$. In other words, there exists a matrix $M_{0} \in \mathcal{M}(X, Y)$ such that $\mathbf{s}\left(M_{0}\right)=\mathbf{b}$.

Proof. Assume that $\mathbf{b} \notin \mathcal{L}(X, Y)$. Since $\mathcal{L}(X, Y)$ is a closed convex set it follows from the Hahn-Banach theorem that there exist $c \in \mathbb{R}$ and $\left(r_{1}, \ldots, r_{n}\right) \in \mathbb{R}^{n}$ such that

$$
\sum_{j=1}^{n} r_{j} s_{j}<c<\sum_{j=1}^{n} r_{j} b_{j} \text { for }\left(s_{1}, \ldots, s_{n}\right) \in \mathcal{L}(X, Y) .
$$

Given an arbitrary vector $\mathbf{y} \in \operatorname{conv}\left(\mathbf{y}_{1}, \ldots, \mathbf{y}_{n}\right)$ we define the simplex

$$
W(\mathbf{y})=\left\{\left(\lambda_{1}, \ldots, \lambda_{n}\right) \in[0,1]^{n} \mid \mathbf{y}=\sum_{j=1}^{n} \lambda_{j} \mathbf{y}_{j}, \sum_{j=1}^{n} \lambda_{j}=1\right\} .
$$

Note that $W(\mathbf{y})$ is compact for any $\mathbf{y} \in \operatorname{conv}\left(\mathbf{y}_{1}, \ldots, \mathbf{y}_{n}\right)$ since it is complete and totally bounded. We may therefore define a function $G: \operatorname{conv}\left(\mathbf{y}_{1}, \ldots, \mathbf{y}_{n}\right) \rightarrow \mathbb{R}$ by setting

$$
G(\mathbf{y})=\max \left\{\sum_{j=1}^{n} r_{j} \lambda_{j} \mid\left(\lambda_{1}, \ldots, \lambda_{n}\right) \in W(\mathbf{y})\right\} \text { for } \mathbf{y} \in \operatorname{conv}\left(\mathbf{y}_{1}, \ldots, \mathbf{y}_{n}\right) .
$$

Let $\lambda_{j}(\mathbf{y}) \in[0,1], 1 \leq j \leq n$, be such that $G(\mathbf{y})=\sum_{j=1}^{n} r_{j} \lambda_{j}(\mathbf{y})$. Since $\lambda_{j}\left(\mathbf{y}_{i}\right)=\delta_{i j}$ one has $G\left(\mathbf{y}_{j}\right) \geq r_{j}$ for $1 \leq j \leq n$ and thus

$$
\sum_{j=1}^{n} b_{j} G\left(\mathbf{y}_{j}\right) \geq \sum_{j=1}^{n} b_{j} r_{j}>c
$$

On the other hand the left inequality in (2.3) implies that for any matrix $M=$ $\left(m_{i j}\right) \in \mathcal{M}(X, Y)$ one has

$$
c>\sum_{j=1}^{n} r_{j} s_{j}(M)=\sum_{i=1}^{m} \sum_{j=1}^{n} a_{i} r_{j} m_{i j} .
$$

Let us now consider the extension $\tilde{G}$ of the function $G$ to $\mathbb{R}^{k}$ given by $\tilde{G}(\mathbf{y})=$ $G(\mathbf{y})$ if $\mathbf{y} \in \operatorname{conv}\left(\mathbf{y}_{1}, \ldots, \mathbf{y}_{n}\right)$ and $\tilde{G}(\mathbf{y})=0$ for $\mathbf{y} \in \mathbb{R}^{k} \backslash \operatorname{conv}\left(\mathbf{y}_{1}, \ldots, \mathbf{y}_{n}\right)$. It is not difficult to see that $\tilde{G}$ is a concave function on $\mathbb{R}^{k}$. By Lemma 1 one has $\mathbf{x}_{i} \in \operatorname{conv}\left(\mathbf{y}_{1}, \ldots, \mathbf{y}_{n}\right), 1 \leq i \leq m$, so that the numbers $\lambda_{j}\left(\mathbf{x}_{i}\right)$ are well defined for 
$1 \leq i \leq m, 1 \leq j \leq n$. Note that $\left(\lambda_{j}\left(\mathbf{x}_{i}\right)\right) \in \mathcal{M}(X, Y)$ and that by (2.5) and (2.4) the following holds:

$$
\sum_{i=1}^{m} a_{i} \tilde{G}\left(\mathbf{x}_{i}\right)=\sum_{i=1}^{m} \sum_{j=1}^{n} a_{i} r_{j} \lambda_{j}\left(\mathbf{x}_{i}\right)<\sum_{j=1}^{n} b_{j} r_{j} \leq \sum_{j=1}^{n} b_{j} \tilde{G}\left(\mathbf{y}_{j}\right) .
$$

This however contradicts condition (i) of Theorem 1 and thus we are done. It follows that $\mathbf{b} \in \mathcal{L}(X, Y)$, which completes the proof of the lemma.

The fact that (ii) $\Rightarrow$ (i) in Theorem 1 is equivalent to Lemma 2 ,

Remark 1 . The authors are grateful to the referee for pointing out the similarity between the Hahn-Banach type argument used above and the one given in the original proof of the Cartier-Fell-Meyer theorem [7].

Definition 1. The pair $(X, \mathbf{a}) \in \mathbb{X}_{m}^{k}$ is said to be weightily majorized by the pair $(Y, \mathbf{b}) \in \mathbb{X}_{n}^{k}$, denoted $(X, \mathbf{a}) \prec(Y, \mathbf{b})$, if the conditions of Theorem 1 are satisfied.

Remark 2. If $(X, \mathbf{a}) \prec(Y, \mathbf{b})$, then the a-barycenter of $X$ must coincide with the b-barycenter of $Y$, that is, $\sum_{i=1}^{m} a_{i} \mathbf{x}_{i}=\sum_{j=1}^{n} b_{j} \mathbf{y}_{j}$.

It is clear from Definition 1 that the weighted majorization relation is a preorder on $\mathbb{X}^{k}$. Indeed, by Theorem 1 this relation is both reflexive and transitive. Moreover, if $m=n$ and $a_{i}=b_{j}=\frac{1}{n}, 1 \leq i, j \leq n$, then Theorem 1 and Birkhoff's theorem [27, Theorem A.2] imply that the weighted multivariate majorization relation induces a partial ordering on the set of unordered $n$-tuples of vectors in $\mathbb{R}^{k}$. We shall refer to this partial ordering as the ordinary multivariate majorization relation, for which Sherman's theorem takes a particularly simple form:

Corollary 1. If $X=\left(\mathbf{x}_{1}, \ldots, \mathbf{x}_{n}\right) \in \mathcal{X}_{n}^{k}$ and $Y=\left(\mathbf{y}_{1}, \ldots, \mathbf{y}_{n}\right) \in \mathcal{X}_{n}^{k}$, then the following conditions are equivalent:

(i) The inequality $\sum_{i=1}^{n} \Phi\left(\mathbf{x}_{i}\right) \leq \sum_{j=1}^{n} \Phi\left(\mathbf{y}_{j}\right)$ holds for any convex function $\Phi: \mathbb{R}^{k} \rightarrow \mathbb{R}$.

(ii) There exists a doubly stochastic $n \times n$ matrix $S$ such that $\tilde{X}^{T}=S \tilde{Y}^{T}$, where $\tilde{X}^{T}$ and $\tilde{Y}^{T}$ are $n \times k$ matrices obtained by some ordering of the vectors in $X^{T}$ and $Y^{T}$, respectively.

Remark 3. For $k=1$ Corollary 1 amounts to a well-known description of classical (vector) majorization due to Schur and to Hardy-Littlewood-Pólya [19, 27.

Remark 4 . In the sequel we shall often consider weighted pairs of the form $(X, \mathbf{a})$ and $(Y, \mathbf{b})$, where $X$ and $Y$ are unordered tuples of complex numbers. In this case we define the weighted majorization relation $(X, \mathbf{a}) \prec(Y, \mathbf{b})$, when appropriate, by identifying $\mathbb{C}$ with $\mathbb{R}^{2}$ in Theorem 1 and Definition 1 .

Another interesting consequence of Sherman's theorem is as follows:

Corollary 2. Assume that $m<n \leq k$ and let $\left(\mathbf{x}_{1}, \ldots, \mathbf{x}_{m}\right) \in \mathcal{X}_{m}^{k},\left(\mathbf{y}_{1}, \ldots, \mathbf{y}_{n}\right) \in$ $\mathcal{X}_{n}^{k},\left(a_{1}, \ldots, a_{n}\right) \in \mathcal{A}_{n}$ and $\left(b_{1}, \ldots, b_{n}\right) \in \mathcal{A}_{n}$. For $m+1 \leq i \leq n$ let $\left(c_{i 1}, \ldots, c_{i n}\right) \in$ $\mathcal{A}_{n}$ be such that $b_{j}^{\prime}:=b_{j}-\sum_{i=m+1}^{n} a_{i} c_{i j} \neq 0,1 \leq j \leq n$, and set $\mathbf{x}_{i}=\sum_{j=1}^{n} c_{i j} \mathbf{y}_{j}$. If $\mathbf{y}_{1}, \ldots, \mathbf{y}_{n}$ are linearly independent, then the following conditions are equivalent:

(i) $\sum_{i=1}^{n} a_{i} \Phi\left(\mathbf{x}_{i}\right) \leq \sum_{j=1}^{n} b_{j} \Phi\left(\mathbf{y}_{j}\right)$ for all convex functions $\Phi: \mathbb{R}^{k} \rightarrow \mathbb{R}$.

(ii) $\sum_{i=1}^{m} a_{i} \Phi\left(\mathbf{x}_{i}\right) \leq \sum_{j=1}^{n} b_{j}^{\prime} \Phi\left(\mathbf{y}_{j}\right)$ for all convex functions $\Phi: \mathbb{R}^{k} \rightarrow \mathbb{R}$.

If either of these conditions is fulfilled, then $b_{j}^{\prime}>0$ for $1 \leq j \leq n$. 
Proof. The implication (ii) $\Rightarrow$ (i) being trivial let us show that (i) $\Rightarrow$ (ii). Set $X=\left(\mathbf{x}_{1}, \ldots, \mathbf{x}_{n}\right) \in \mathcal{X}_{n}^{k}, X^{\prime}=\left(\mathbf{x}_{1}, \ldots, \mathbf{x}_{m}\right), Y=\left(\mathbf{y}_{1}, \ldots, \mathbf{y}_{n}\right), \mathbf{a}=\left(a_{1}, \ldots, a_{n}\right)$ and $\mathbf{b}=\left(b_{1}, \ldots, b_{n}\right)$. Then condition (i) is equivalent to $(X, \mathbf{a}) \prec(Y, \mathbf{b})$. Note that $\sum_{j=1}^{n} b_{j}^{\prime}=\sum_{i=1}^{m} a_{i}=: \alpha \in(0,1)$ and let $\mathbf{a}^{\prime}=\left(\alpha^{-1} a_{1}, \ldots, \alpha^{-1} a_{m}\right) \in \mathcal{A}_{m}$ and $\mathbf{b}^{\prime}=\left(\alpha^{-1} b_{1}^{\prime}, \ldots, \alpha^{-1} b_{n}^{\prime}\right)$. Using Theorem 1 and the fact that $\mathbf{y}_{1}, \ldots, \mathbf{y}_{n}$ are linearly independent one can easily show that $\mathbf{b}^{\prime} \in \mathcal{A}_{n}$ and $\left(X^{\prime}, \mathbf{a}^{\prime}\right) \prec\left(Y, \mathbf{b}^{\prime}\right)$, which is equivalent to condition (ii).

Remark 5. For an arbitrarily given convex function $\Phi: \mathbb{R}^{k} \rightarrow \mathbb{R}$ the inequality in Corollary 2 (i) is à priori weaker than the one in (ii). As a special case let us consider three complex numbers $z_{1}, z_{2}, z_{3}$ with $z_{2} \bar{z}_{3} \notin \mathbb{R}$ and $\alpha, \beta, \gamma \in(0,1)$ such that $\gamma \neq(1-\alpha) \beta$ and $1-\gamma \neq(1-\alpha)(1-\beta)$. Then Corollary 2 implies that if

$$
\alpha \Phi\left(z_{1}\right)+(1-\alpha) \Phi\left(\beta z_{2}+(1-\beta) z_{3}\right) \leq \gamma \Phi\left(z_{2}\right)+(1-\gamma) \Phi\left(z_{3}\right)
$$

for all convex functions $\Phi: \mathbb{C} \rightarrow \mathbb{R}$, then $\gamma>(1-\alpha) \beta, 1-\gamma>(1-\alpha)(1-\beta)$, and the inequality

$$
\alpha \Phi\left(z_{1}\right)+(1-\alpha)\left(\beta \Phi\left(z_{2}\right)+(1-\beta) \Phi\left(z_{3}\right)\right) \leq \gamma \Phi\left(z_{2}\right)+(1-\gamma) \Phi\left(z_{3}\right)
$$

holds for any convex function $\Phi: \mathbb{C} \rightarrow \mathbb{R}$.

Remark 6. Theorem 1 generalizes several known results on vector and matrix majorization, many of which seem in fact to be regularly rediscovered in various contexts; see, e.g., 9 and references therein. Two new interesting extensions of classical and multivariate majorization have recently been introduced and studied in [26].

\section{Geometry of equilibrium points: Statement of main Results}

In this section we formulate our main results. These describe the distribution of equilibrium points of logarithmic potentials for discrete configurations of positive charges (Theorems 2, 3 and Theorems 647) and the Hausdorff geometry of the extended set of equilibrium points for finite charge configurations (Theorems (45).

3.1. Weighted majorization relations for finite charge configurations. Let $z_{i}, 1 \leq i \leq n$, be distinct points in the complex plane carrying positive charges $a_{i}$, $1 \leq i \leq n$, respectively, where $n \geq 2$. Throughout this section we assume wlog that the $a_{i}$ are normalized so that $\sum_{i=1}^{n} a_{i}=1$. The logarithmic potential generated by these charged particles is given by

$$
U(z)=\sum_{i=1}^{n} a_{i} \log \left|1-\frac{z}{z_{i}}\right|,
$$

and the resulting electrostatic force is

$$
f(z)=\sum_{i=1}^{n} \frac{a_{i}}{z-z_{i}} .
$$

The class of functions of the form (3.2) is essentially the same as Sz-Nagy's class of generalized derivatives [32. As explained in $\$ 1$, the critical points of $U(z)$ coincide with the equilibrium points of the electrostatic field, that is, the zeros of $f(z)$. By an argument reminiscent of the Gauss-Lucas theorem one can see that all equilibrium points lie in $\operatorname{conv}\left(z_{1}, \ldots, z_{n}\right)$; cf., e.g., Proposition 3.1 in [26]. Remarkable generalizations of this simple fact were recently obtained in loc. cit., where it was 
shown that these points actually satisfy a whole sequence of majorization relations. Theorems 23 below further extend the aforementioned results.

Notation 2. Let $w_{1}, \ldots, w_{n-1}$ be the critical points of $U(z)$ counted with multiplicity and recall the set $\mathcal{A}_{m}$ defined in (2.1). For $\lambda \in \mathbb{C} \backslash\{0\}$ and $\mu \in \mathbb{C}$ let

$$
\begin{aligned}
& W(\lambda, \mu)=\left(\lambda w_{1}+\mu, \ldots, \lambda w_{n-1}+\mu\right) \in \mathbb{C}^{n-1} \\
& Z(\lambda, \mu)=\left(\lambda z_{1}+\mu, \ldots, \lambda z_{n}+\mu\right) \in \mathbb{C}^{n} \\
& \mathbf{a}=\left(\frac{1}{n-1}, \ldots, \frac{1}{n-1}\right) \in \mathcal{A}_{n-1}, \quad \mathbf{b}=\left(\frac{1-a_{1}}{n-1}, \ldots, \frac{1-a_{n}}{n-1}\right) \in \mathcal{A}_{n}
\end{aligned}
$$

Theorem 2. There exists a matrix $R \in \mathbb{M}_{n-1, n}^{r s}$ such that $W(\lambda, \mu)^{T}=R Z(\lambda, \mu)^{T}$ and $\mathbf{b}=\mathbf{a} R$ for any $\lambda \in \mathbb{C} \backslash\{0\}$ and $\mu \in \mathbb{C}$, so that $(W(\lambda, \mu), \mathbf{a}) \prec(Z(\lambda, \mu), \mathbf{b})$. Equivalently, the inequality

$$
\sum_{j=1}^{n-1} \Phi\left(\lambda w_{j}+\mu\right) \leq \sum_{i=1}^{n}\left(1-a_{i}\right) \Phi\left(\lambda z_{i}+\mu\right)
$$

is satisfied by any (continuous) convex function $\Phi: \mathbb{C} \rightarrow \mathbb{R}$.

Remark 7. The special case of (3.4) when $\lambda=1, \mu=0$ and $a_{i}=\frac{1}{n}, 1 \leq i \leq n$, is already a considerable improvement of the Gauss-Lucas theorem and was originally conjectured by de Bruijn and Springer [6]. This conjecture was recently proved by S. Malamud 25, 26] and R. Pereira 28] independently of each other.

Remark 8. Theorem2 2 implies in particular that the following ordinary multivariate majorization relation holds in $\mathbb{R}^{2}$ :

$$
\left(w_{1}, \ldots, w_{n-1}, \zeta\right) \prec\left(z_{1}, \ldots, z_{n-1}, z_{n}\right),
$$

where $\zeta=\sum_{i=1}^{n} a_{i} z_{i}$ is the $\left(a_{1}, \ldots, a_{n}\right)$-barycenter of $Z(1,0)$ (cf. Remarks 2 and (4). This relation was previously obtained in [26, Proposition 4.3] and [28, Theorem 5.4]. Note though that if $n \geq 3$, then even in the case of equal charges the above relation is in general weaker than the corresponding one given by Theorem 2 since the $z_{i}$ are linearly dependent (compare with Corollary 2).

As we shall now see, (3.4) is actually but one relation in a sequence of $\left(\begin{array}{l}n \\ 2\end{array}\right)$ weighted multivariate majorization relations satisfied by the zeros and poles of $f(z)$. Moreover, the equilibrium points of $U(z)$ are in fact uniquely determined by $n-1$ such relations. To formulate these results we need some new notation.

Notation 3. Given $k \in \mathbb{N}$ and $1 \leq m \leq k$ let $\Pi_{k, m}$ denote the $m$-th elementary symmetric function on $k$ symbols. For $\lambda \in \mathbb{C} \backslash\{0\}, \mu \in \mathbb{C}, k \in\{1, \ldots, n-1\}$, and 
$1 \leq m \leq k$ set

$$
\begin{aligned}
& W_{m}^{[k]}(\lambda, \mu)=\left(\omega_{m}\left[r_{1}, \ldots, r_{k}\right](\lambda, \mu)\right)_{1 \leq r_{1}<\ldots<r_{k} \leq n-1} \in \mathbb{C}^{\left(\begin{array}{c}
n-1 \\
k
\end{array}\right),}, \\
& \text { where } \omega_{m}\left[r_{1}, \ldots, r_{k}\right](\lambda, \mu)=\Pi_{k, m}\left(\lambda w_{r_{1}}+\mu, \ldots, \lambda w_{r_{k}}+\mu\right), \\
& Z_{m}^{[k]}(\lambda, \mu)=\left(\zeta_{m}\left[s_{1}, \ldots, s_{k}\right](\lambda, \mu)\right)_{1 \leq s_{1}<\ldots<s_{k} \leq n} \in \mathbb{C}^{\left(\begin{array}{c}
n \\
k
\end{array}\right)},
\end{aligned}
$$

where $\zeta_{m}\left[s_{1}, \ldots, s_{k}\right](\lambda, \mu)=\Pi_{k, m}\left(\lambda z_{s_{1}}+\mu, \ldots, \lambda z_{s_{k}}+\mu\right)$,

$$
\begin{aligned}
& \mathbf{a}^{[k]}=\left(\left(\begin{array}{c}
n-1 \\
k
\end{array}\right)^{-1}, \ldots,\left(\begin{array}{c}
n-1 \\
k
\end{array}\right)^{-1}\right) \in \mathcal{A}_{\left(\begin{array}{c}
n-1 \\
k
\end{array}\right)}, \\
& \mathbf{b}^{[k]}=\left(\left(\begin{array}{c}
n-1 \\
k
\end{array}\right)^{-1}\left(1-\sum_{i=1}^{k} a_{s_{i}}\right)\right)_{1 \leq s_{1}<\ldots<s_{k} \leq n} \in \mathcal{A}_{\left(\begin{array}{c}
n \\
k
\end{array}\right)} .
\end{aligned}
$$

Below we shall always assume that the components of $W_{m}^{[k]}(\lambda, \mu), Z_{m}^{[k]}(\lambda, \mu)$ and $\mathbf{b}^{[k]}$ are arranged increasingly with respect to the lexicographic order on the set of all indices consisting of $k$-tuples of increasing numbers in $\{1, \ldots, n\}$. In particular, for any $1 \leq m \leq\left(\begin{array}{c}n \\ k\end{array}\right)$ the $m$-th coordinates of $Z_{m}^{[k]}(\lambda, \mu)$ and $\mathbf{b}^{[k]}$ are indexed by the same $k$-tuple $\left(s_{1}, \ldots, s_{k}\right)$ satisfying $1 \leq s_{1}<\ldots<s_{k} \leq n$. Note also that with this ordering (3.3) and (3.5) imply that $\mathbf{a}^{[1]}=\mathbf{a}, \mathbf{b}^{[1]}=\mathbf{b}, W_{1}^{[1]}(\lambda, \mu)=W(\lambda, \mu)$ and $Z_{1}^{[1]}(\lambda, \mu)=Z(\lambda, \mu)$.

Theorem 3.11 in [26] describes all possible sets of equilibrium points for logarithmic potentials generated by a fixed configuration of particles that are allowed to carry arbitrary positive charges. A natural related inverse problem is to describe geometrically the equilibrium points of a logarithmic potential associated to a given set of particles with prescribed positive charges. The following theorem solves this problem and provides a characterization of the zeros of the logarithmic potential $U(z)$ defined in (3.1) by means of $n-1$ weighted majorization relations.

Theorem 3. The following conditions are equivalent:

(i) The zeros of $f(z)$ counted with multiplicity are $w_{1}, \ldots, w_{n-1}$, that is,

$$
\sum_{i=1}^{n} a_{i} \prod_{\substack{j=1 \\ j \neq i}}^{n}\left(z-z_{j}\right)=\prod_{k=1}^{n-1}\left(z-w_{k}\right), \quad z \in \mathbb{C}
$$

(ii) There exist $\lambda \in \mathbb{C} \backslash\{0\}$ and $\mu \in \mathbb{C}$ such that

$$
\left(W_{k}^{[k]}(\lambda, \mu), \mathbf{a}^{[k]}\right) \prec\left(Z_{k}^{[k]}(\lambda, \mu), \mathbf{b}^{[k]}\right), \quad 1 \leq k \leq n-1 .
$$

(iii) There exist $\lambda \in \mathbb{C} \backslash\{0\}$ and $\mu \in \mathbb{C}$ such that for any $k \in\{1, \ldots, n-1\}$ one can find an $\left(\begin{array}{c}n-1 \\ k\end{array}\right) \times\left(\begin{array}{l}n \\ k\end{array}\right)$ row stochastic matrix $R_{k}(\lambda, \mu)$ satisfying

$$
W_{k}^{[k]}(\lambda, \mu)^{T}=R_{k}(\lambda, \mu) Z_{k}^{[k]}(\lambda, \mu)^{T} \text { and } \mathbf{b}^{[k]}=\mathbf{a}^{[k]} R_{k}(\lambda, \mu) .
$$


(iv) There exist $\lambda \in \mathbb{C} \backslash\{0\}$ and $\mu \in \mathbb{C}$ such that for any $k \in\{1, \ldots, n-1\}$ the inequality

$$
\begin{aligned}
\sum_{1 \leq r_{1}<\ldots<r_{k} \leq n-1} \Phi\left(\omega_{k}\left[r_{1}, \ldots, r_{k}\right](\lambda, \mu)\right) \\
\leq \sum_{1 \leq s_{1}<\ldots<s_{k} \leq n}\left(1-\sum_{i=1}^{k} a_{s_{i}}\right) \Phi\left(\zeta_{k}\left[s_{1}, \ldots, s_{k}\right](\lambda, \mu)\right)
\end{aligned}
$$

holds for all (continuous) convex functions $\Phi: \mathbb{C} \rightarrow \mathbb{R}$.

(v) Either of conditions (ii)-(iv) is true for $\lambda=1$ and $\mu=0$.

If the above conditions are fulfilled, then for any $k \in\{1, \ldots, n-1\}$ there exists an $\left(\begin{array}{c}n-1 \\ k\end{array}\right) \times\left(\begin{array}{l}n \\ k\end{array}\right)$ row stochastic matrix $R_{k}$ such that

$$
W_{k}^{[k]}(\lambda, \mu)^{T}=R_{k} Z_{k}^{[k]}(\lambda, \mu)^{T} \text { and } \mathbf{b}^{[k]}=\mathbf{a}^{[k]} R_{k}
$$

for all $\lambda \in \mathbb{C} \backslash\{0\}$ and $\mu \in \mathbb{C}$.

Remark 9. Theorem 3 extends [26, Theorem 4.7] to the case of arbitrary (not necessarily equal) charges. If $a_{i}=\frac{1}{n}, 1 \leq i \leq n$, then Theorem 3 reduces to the characterization of the zeros and critical points of degree $n$ complex polynomials by means of $n-1$ majorization relations which was given in [26, Theorem 4.10].

From Theorem 3 we deduce a number of corollaries.

Corollary 3. For any $k \in\{1, \ldots, n-1\}$ there exists an $\left(\begin{array}{c}n-1 \\ k\end{array}\right) \times\left(\begin{array}{l}n \\ k\end{array}\right)$ row stochastic matrix $R_{k}$ such that

$$
W_{m}^{[k]}(\lambda, \mu)^{T}=R_{k} Z_{m}^{[k]}(\lambda, \mu)^{T} \text { and } \mathbf{b}^{[k]}=\mathbf{a}^{[k]} R_{k}
$$

whenever $1 \leq m \leq k, \lambda \in \mathbb{C} \backslash\{0\}$ and $\mu \in \mathbb{C}$. In particular, for all such parameters $k, m, \lambda, \mu$ and any convex function $\Phi: \mathbb{C} \rightarrow \mathbb{R}$ the following inequality holds:

$$
\begin{aligned}
\sum_{1 \leq r_{1}<\ldots<r_{k} \leq n-1} \Phi\left(\omega_{m}\left[r_{1}, \ldots, r_{k}\right](\lambda, \mu)\right) \\
\leq \sum_{1 \leq s_{1}<\ldots<s_{k} \leq n}\left(1-\sum_{i=1}^{k} a_{s_{i}}\right) \Phi\left(\zeta_{m}\left[s_{1}, \ldots, s_{k}\right](\lambda, \mu)\right) .
\end{aligned}
$$

Remark 10. Note that (3.8) is à priori stronger than (3.9) since it asserts that there exist matrices $R_{k}$ of the desired type that simultaneously realize the weighted majorization relations between the pairs $\left(W_{m}^{[k]}(\lambda, \mu), \mathbf{a}^{[k]}\right)$ and $\left(Z_{m}^{[k]}(\lambda, \mu), \mathbf{b}^{[k]}\right)$ for all admissible parameters $m, \lambda$ and $\mu$.

Setting $m=1$ in Corollary 3 yields the following generalization of Theorem 2 , which corresponds to the case when $k=1$ in (3.10) below.

Corollary 4. For any $k \in\{1, \ldots, n-1\}$ there exists an $\left(\begin{array}{c}n-1 \\ k\end{array}\right) \times\left(\begin{array}{c}n \\ k\end{array}\right)$ row stochastic matrix $R_{k}$ such that

$$
W_{1}^{[k]}(\lambda, \mu)^{T}=R_{k} Z_{1}^{[k]}(\lambda, \mu)^{T} \text { and } \mathbf{b}^{[k]}=\mathbf{a}^{[k]} R_{k}
$$

for any $\lambda \in \mathbb{C} \backslash\{0\}$ and $\mu \in \mathbb{C}$. Thus

$$
\left(W_{1}^{[k]}(\lambda, \mu), \mathbf{a}^{[k]}\right) \prec\left(Z_{1}^{[k]}(\lambda, \mu), \mathbf{b}^{[k]}\right), \quad 1 \leq k \leq n-1,
$$


or equivalently, for all convex functions $\Phi: \mathbb{C} \rightarrow \mathbb{R}$ and $k \in\{1, \ldots, n-1\}$ one has

$$
\begin{aligned}
\sum_{1 \leq r_{1}<\ldots<r_{k} \leq n-1} & \Phi\left(\omega_{1}\left[r_{1}, \ldots, r_{k}\right](\lambda, \mu)\right) \\
\leq & \sum_{1 \leq s_{1}<\ldots<s_{k} \leq n}\left(1-\sum_{i=1}^{k} a_{s_{i}}\right) \Phi\left(\zeta_{1}\left[s_{1}, \ldots, s_{k}\right](\lambda, \mu)\right) .
\end{aligned}
$$

Remark 11. The condition $\lambda \neq 0$ may actually be omitted from the above inequalities. However, if $\lambda=0$ the vectors defined in (3.5) are independent of the $z_{i}$ and $w_{j}$, and the aforementioned inequalities become equalities. Thus the corresponding weighted majorization relations are trivially satisfied in this case.

Taking $\lambda=1, \mu=0$ and $\Phi(z)=|z|^{\alpha}$ with $\alpha \geq 1$ in Corollary 3 we obtain the following moment inequalities for the charged particles and the equilibrium points.

Corollary 5. For any $k \in\{1, \ldots, n-1\}, 1 \leq m \leq k$, and $\alpha \geq 1$ one has

$$
\begin{aligned}
\sum_{1 \leq r_{1}<\ldots<r_{k} \leq n-1}\left|\Pi_{k, m}\left(w_{r_{1}}, \ldots, w_{r_{k}}\right)\right|^{\alpha} \\
\leq \sum_{1 \leq s_{1}<\ldots<s_{k} \leq n}\left|\Pi_{k, m}\left(z_{s_{1}}, \ldots, z_{s_{k}}\right)\right|^{\alpha},
\end{aligned}
$$

where $\Pi_{k, m}$ denotes as before the $m$-th elementary symmetric function on $k$ symbols.

In particular, by setting $a_{i}=\frac{1}{n}, 1 \leq i \leq n$, and $m=1$ in Corollary [5 we get

$$
\left(\begin{array}{c}
n-1 \\
k
\end{array}\right)^{-1} \sum_{1 \leq r_{1}<\ldots<r_{k} \leq n-1}\left|\sum_{i=1}^{k} w_{r_{i}}\right|^{\alpha} \leq\left(\begin{array}{c}
n \\
k
\end{array}\right)^{-1} \sum_{1 \leq s_{1}<\ldots<s_{k} \leq n}\left|\sum_{i=1}^{k} z_{s_{i}}\right|^{\alpha}
$$

for all $\alpha \geq 1$ and $k \in\{1, \ldots, n-1\}$. Note that for $k=2$ and $\alpha \geq 1$ the inequalities in (3.11) are quite similar to the inequalities

$$
\left(\begin{array}{c}
n-1 \\
2
\end{array}\right)^{-1} \sum_{1 \leq r_{1}<r_{2} \leq n-1}\left|w_{r_{1}}-w_{r_{2}}\right|^{\alpha} \leq\left(\begin{array}{c}
n \\
2
\end{array}\right)^{-1} \sum_{1 \leq s_{1}<s_{2} \leq n}\left|z_{s_{1}}-z_{s_{2}}\right|^{\alpha}
$$

that were conjectured by Rahman and Schmeisser in [30, Remark 2.3.9]. In $\$ 5$ we conjecture a whole hierarchy of weighted de Bruijn-Springer relations and inertia laws; see Conjecture 1 below. The latter actually contains as special cases all types of moment inequalities like those that we just described.

3.2. Relative Hausdorff geometry. The metric function between (closed) subsets of Euclidean space introduced by Hausdorff makes use of the following notions.

Definition 2. The relative (alternatively, directed or oriented) Hausdorff distance from a subset $\Omega_{1}$ of $\mathbb{C}$ to another such subset $\Omega_{2}$ is given by

$$
h\left(\Omega_{1}, \Omega_{2}\right)=\sup _{w \in \Omega_{1}} \operatorname{dist}\left(w, \Omega_{2}\right)=\sup _{w \in \Omega_{1}} \inf _{z \in \Omega_{2}}|w-z| .
$$

Other frequent names and notations are the forward Hausdorff distance from $\Omega_{1}$ to $\Omega_{2}, h_{f}\left(\Omega_{1}, \Omega_{2}\right):=h\left(\Omega_{1}, \Omega_{2}\right)$, and the backward Hausdorff distance from $\Omega_{1}$ to $\Omega_{2}$, $h_{b}\left(\Omega_{1}, \Omega_{2}\right):=h\left(\Omega_{2}, \Omega_{1}\right)$. The (symmetrized) Hausdorff distance between $\Omega_{1}$ and $\Omega_{2}$ is then given by

$$
H\left(\Omega_{1}, \Omega_{2}\right)=\max \left(h\left(\Omega_{1}, \Omega_{2}\right), h\left(\Omega_{2}, \Omega_{1}\right)\right) .
$$


Let $\mathcal{Z}=\left\{z_{1}, \ldots, z_{n}\right\}$ be a set of charged particles and $\mathcal{W}=\left\{w_{1}, \ldots, w_{n-1}\right\}$ be the corresponding set of equilibrium points of the logarithmic potential defined in (3.1). Theorems 2 3yield a series of relations between the sets $\mathcal{W}$ and $\mathcal{Z}$ when all their elements are considered simultaneously. These relations go much beyond the obvious inclusion $\mathcal{W} \subset \operatorname{conv}(\mathcal{Z})$ and suggest that no equilibrium point can actually lie too far away from the particles. Our next result gives a precise meaning to this intuitive fact and shows that the relative Hausdorff distance $h(\mathcal{W}, \mathcal{Z})$ is dominated by the a-weighted standard deviation of the particles. The latter is given by the square root of the a-weighted variance of $\mathbf{z}=\left(z_{1}, \ldots, z_{n}\right)$, which we define as

$$
\sigma_{2}(\mathbf{z} ; \mathbf{a})^{2}=\inf _{\alpha \in \mathbb{C}} \sum_{i=1}^{n} a_{i}\left|z_{i}-\alpha\right|^{2}=\sum_{i=1}^{n} a_{i}\left|z_{i}-\zeta\right|^{2},
$$

where $\mathbf{a}=\left(a_{1}, \ldots, a_{n}\right) \in \mathcal{A}_{n}$ and $\zeta$ stands as before for the a-weighted barycenter $\sum_{i=1}^{n} a_{i} z_{i}$ (cf. Remark 8). We shall actually prove a stronger statement involving the extended set of equilibrium points of the given system of positive charges, i.e., the set

$$
\mathcal{W}_{e}=\mathcal{W} \cup\{\zeta\}=\left\{w_{1}, \ldots, w_{n-1}, \zeta\right\} .
$$

Theorem 4. In the above notation one $h a s h(\mathcal{W}, \mathcal{Z}) \leq h\left(\mathcal{W}_{e}, \mathcal{Z}\right) \leq \sigma_{2}(\mathbf{z} ; \mathbf{a})$.

Remark 12. The bound given in Theorem 4 is sharp, as one can see for instance by placing $n$ equal charges at the vertices of a regular $n$-gon.

A natural question in this context is whether there is any analog of Theorem 4 for the relative Hausdorff distance from $\mathcal{Z}$ to $\mathcal{W}_{e}$. The following theorem provides an affirmative answer to this question when all particles are assumed to lie on the same line and shows that in this case the Hausdorff geometry of the set of charged particles and the extended set of equilibrium points is controlled by the weighted standard deviation of the particles.

Theorem 5. If $z_{1}, \ldots, z_{n}$ are collinear, then $H\left(\mathcal{Z}, \mathcal{W}_{e}\right) \leq \sigma_{2}(\mathbf{z} ; \mathbf{a})$.

Remark 13. Note that Theorem 5 is sharp and that a similar result cannot hold if $\mathcal{W}_{e}$ is replaced by $\mathcal{W}$. To see this one may simply consider two distinct points in the plane carrying unequal positive charges.

3.3. Infinite discrete distributions of charge. As we point out in 86 , several difficulties occur when trying to extend the results of 3.1 to logarithmic potentials generated by an infinite discrete set of positive charges. To begin with, in this case the fundamental question dealing with the existence of equilibrium points is already quite delicate and, as a matter of fact, still an open problem (cf. [8, 14, 24]).

Nevertheless, it turns out that in many situations one can obtain new information on the geometry of the electrostatic field through natural infinite-dimensional versions of Theorem 2 As we shall now explain, this is for instance the case for collinear positive charges and, more generally, for essentially any bounded discrete set of positively charged particles with finite total charge for which equilibrium points are known to exist. Let $\left\{a_{i}\right\}_{i \in \mathbb{N}}$ be a sequence of positive numbers and let $\left\{z_{i}\right\}_{i \in \mathbb{N}}$ be a sequence of distinct complex numbers satisfying the conditions

$$
a_{i}>0 \text { and }\left|z_{i}\right|<\rho \text { for } i \in \mathbb{N}, \quad \sum_{i=1}^{\infty} a_{i}=1, \quad \lim _{i \rightarrow \infty} z_{i}=\rho,
$$


where $\rho$ is a fixed positive number. Using the same electrostatic interpretation as before - that is, placing a charge $a_{i}$ at $z_{i}$ for $i \in \mathbb{N}$ - we get the resulting force

$$
f(z)=\sum_{i=1}^{\infty} \frac{a_{i}}{z-z_{i}}
$$

and an associated logarithmic potential given by

$$
U(z)=\sum_{i=1}^{\infty} a_{i} \log \left|1-\frac{z}{z_{i}}\right| .
$$

Set $\mathbb{D}(\rho)=\{z \in \mathbb{C}:|z|<\rho\}$ and $\mathbb{D}_{f}(\rho)=\mathbb{D}(\rho) \backslash\left\{z_{i}\right\}_{i \in \mathbb{N}}$. By (3.14) one has

$$
\sum_{i=1}^{\infty} \frac{a_{i}}{\left|z_{i}\right|}<\infty
$$

so that the series in the right-hand side of (3.15) converges absolutely for all $z \in$ $\mathbb{D}_{f}(\rho)$ and thus $f$ is a meromorphic function in $\mathbb{D}(\rho)$. One can see in a similar fashion that $U$ is subharmonic in $\mathbb{D}(\rho)$. We have the following analog of Theorem 2 ,

Theorem 6. If $\left\{z_{i}\right\}_{i \in \mathbb{N}}$ is a real sequence satisfying (3.14), then the function $f$ defined by (3.15) has an infinite discrete set of real zeros $\left\{w_{j}\right\}_{j \in \mathbb{N}}$ and the sequences $\left\{z_{i}\right\}_{i \in \mathbb{N}}$ and $\left\{w_{j}\right\}_{j \in \mathbb{N}}$ interlace on the real axis. Moreover, there exists an infinite matrix $S=\left(s_{i j}\right)_{i, j=1}^{\infty}$ such that for all $(i, j) \in \mathbb{N}^{2}$ one has

$$
s_{i j} \geq 0, \quad \sum_{k=1}^{\infty} s_{i k}=1, \quad \sum_{l=1}^{\infty} s_{l j}=1-a_{j}, \quad w_{i}=\sum_{k=1}^{\infty} s_{i k} z_{k} .
$$

In particular, for any $\lambda \in \mathbb{C} \backslash\{0\}$ and $\mu \in \mathbb{C}$ the inequality

$$
\sum_{j=1}^{\infty} \Phi\left(\lambda w_{j}+\mu\right) \leq \sum_{i=1}^{\infty}\left(1-a_{i}\right) \Phi\left(\lambda z_{i}+\mu\right)
$$

holds for any convex function $\Phi: \mathbb{C} \rightarrow \mathbb{R}$.

Our last main result is a natural analog of Theorem 6 for all bounded discrete configurations of positive charges for which equilibrium points are known to exist.

Theorem 7. Let $\left\{z_{i}\right\}_{i \in \mathbb{N}}$ be a sequence of distinct complex numbers satisfying (3.14) and let $f$ be the meromorphic function in $\mathbb{D}(\rho)$ defined by (3.15). If $f$ has a nonempty (discrete) set of zeros $\left\{w_{j}\right\}_{j \in \mathbb{N}}$ then the inequality

$$
\sum_{j=1}^{\infty} \Phi\left(\lambda w_{j}+\mu\right) \leq \sum_{i=1}^{\infty}\left(1-a_{i}\right) \Phi\left(\lambda z_{i}+\mu\right)
$$

holds for all $\lambda \in \mathbb{C} \backslash\{0\}, \mu \in \mathbb{C}$ and any nonnegative convex function $\Phi: \mathbb{C} \rightarrow \mathbb{R}$.

Remark 14. The fact that inequality (4.18) in $\$ 4.3$ may be strict explains why the inequality in Theorem 7 holds in general only for nonnegative convex functions. The connection between majorization and such inequalities for nonnegative convex functions was first found in [16.

Remark 15. Theorem 7 is reminiscent of Kato's bound for the variation of discrete spectra of Hilbert space selfadjoint operators under compact perturbations 21 . 


\section{Proof of MAIN Results}

4.1. Proof of Theorems 2 2 . We shall use various methods from multilinear algebra and matrix analysis. These require a few notations and preliminary results, and we refer to [1] and [27] for background material.

Notation 4. Given $m \in \mathbb{N}$ and $1 \leq k \leq m$ let

$$
Q_{k, m}=\left\{\mathbf{i}=\left(i_{1}, \ldots, i_{k}\right) \mid 1 \leq i_{1}<\ldots<i_{k} \leq m\right\} .
$$

If $B=\left(b_{i j}\right)$ is an $m \times m$ matrix and $\mathbf{i}, \mathbf{j} \in Q_{k, m}$ we denote by

$$
B(\mathbf{i}, \mathbf{j})=B\left(\begin{array}{l}
i_{1}, \ldots, i_{k} \\
j_{1}, \ldots, j_{k}
\end{array}\right)
$$

the $k \times k$ submatrix of $B$ lying in rows $i_{1}, \ldots, i_{k}$ and columns $j_{1}, \ldots, j_{k}$. Below we shall always assume that the elements of $Q_{k, m}$ are arranged in lexicographic order, i.e., if $\mathbf{i}$ and $\mathbf{j}$ are distinct $k$-tuples in $Q_{k, m}$, then $\mathbf{i} \geq \mathbf{j}$ if the first non-zero term in the sequence $i_{1}-j_{1}, \ldots, i_{k}-j_{k}$ is positive.

We fix an $n$-dimensional complex Hilbert space $\mathcal{H}$ with unitarily invariant scalar product $\langle\cdot, \cdot\rangle$ and identity operator $I=I_{\mathcal{H}}=I_{n} \in L(\mathcal{H})$, where $L(\mathcal{H})$ is the set of all linear operators on $\mathcal{H}$. Let $A \in L(\mathcal{H})$ be a normal operator with spectrum $\Sigma(A)=\left\{z_{1}, \ldots, z_{n}\right\}$ and choose an orthonormal basis $\left(\mathbf{e}_{1}, \ldots, \mathbf{e}_{n}\right)$ of $\mathcal{H}$ such that $A \mathbf{e}_{i}=z_{i} \mathbf{e}_{i}$ for $1 \leq i \leq n$. Define a unit vector

$$
\mathbf{v}_{n}=\sum_{i=1}^{n} \sqrt{a_{i}} \mathbf{e}_{i}
$$

and let $P$ be the orthogonal projection on the subspace $\mathcal{K}:=\mathbf{v}_{n}^{\perp}$ of $\mathcal{H}$. The operator

$$
A^{\prime}=\left.P A P\right|_{\mathcal{K}} \in L(\mathcal{K})
$$

is called the $P$-compression of $A[10]$. Recall the function $f$ defined in (3.2). The following statement may be found in, e.g., [26, formula (3.3)] and [28, Lemma 2.2].

Lemma 3. With the above notations one has

$$
\left\langle\left(A-z I_{\mathcal{H}}\right)^{-1} \mathbf{v}_{n}, \mathbf{v}_{n}\right\rangle=\frac{\operatorname{det}\left(A^{\prime}-z I_{\mathcal{K}}\right)}{\operatorname{det}\left(A-z I_{\mathcal{H}}\right)}=-f(z) \text { for } z \in \mathbb{C} \backslash \Sigma(A) .
$$

Proof. Let $\left(\mathbf{v}_{1}, \ldots, \mathbf{v}_{n-1}\right)$ be an orthonormal basis of $\mathcal{K}$. The matrix representation of $A^{\prime}$ in the basis $\left(\mathbf{v}_{1}, \ldots, \mathbf{v}_{n-1}\right)$ is given by the $(n-1) \times(n-1)$ upper left-hand principal submatrix of the matrix representation of $A$ in the orthonormal basis $\left(\mathbf{v}_{1}, \ldots, \mathbf{v}_{n-1}, \mathbf{v}_{n}\right)$ of $\mathcal{H}$. For any $z \in \mathbb{C} \backslash \Sigma(A)$ the $(n, n)$ entry of the matrix representation of the (normal) operator $\left(A-z I_{\mathcal{H}}\right)^{-1}$ in the basis $\left(\mathbf{v}_{1}, \ldots, \mathbf{v}_{n-1}, \mathbf{v}_{n}\right)$ is given on the one hand by

$$
\left\langle\left(A-z I_{\mathcal{H}}\right)^{-1} \mathbf{v}_{n}, \mathbf{v}_{n}\right\rangle=\left\langle\sum_{i=1}^{n} \frac{\left\langle\mathbf{v}_{n}, \mathbf{e}_{i}\right\rangle}{z_{i}-z} \mathbf{e}_{i}, \sum_{i=1}^{n}\left\langle\mathbf{v}_{n}, \mathbf{e}_{i}\right\rangle \mathbf{e}_{i}\right\rangle=-f(z) .
$$

On the other hand, by Cramer's rule the $(n, n)$ entry of the matrix representation of $\left(A-z I_{\mathcal{H}}\right)^{-1}$ in the basis $\left(\mathbf{v}_{1}, \ldots, \mathbf{v}_{n-1}, \mathbf{v}_{n}\right)$ of $\mathcal{H}$ is given by the cofactor of the $(n, n)$ entry of the matrix representation of $\left(A-z I_{\mathcal{H}}\right)$ in the same basis. Thus

$$
\left\langle\left(A-z I_{\mathcal{H}}\right)^{-1} \mathbf{v}_{n}, \mathbf{v}_{n}\right\rangle=\frac{\operatorname{det}\left(A^{\prime}-z I_{\mathcal{K}}\right)}{\operatorname{det}\left(A-z I_{\mathcal{H}}\right)}
$$

for $z \in \mathbb{C} \backslash \Sigma(A)$, which proves the lemma. 
Proof of Theorem 2. By Lemma 3 the zeros $w_{1}, \ldots, w_{n-1}$ of $f$ are the same as the eigenvalues of the $P$-compression $A^{\prime}$. Let $\left(\mathbf{v}_{1}, \ldots, \mathbf{v}_{n-1}\right)$ be an orthonormal basis of $\mathcal{K}$ that triangularizes $A^{\prime}$. Then

$$
w_{j}=\mathbf{v}_{j}^{*} A^{\prime} \mathbf{v}_{j}=\left\langle A^{\prime} \mathbf{v}_{j}, \mathbf{v}_{j}\right\rangle=\left\langle A \mathbf{v}_{j}, \mathbf{v}_{j}\right\rangle=\sum_{i=1}^{n} z_{i}\left|\left\langle\mathbf{v}_{j}, \mathbf{e}_{i}\right\rangle\right|^{2}
$$

since $\mathbf{v}_{j}=\sum_{i=1}^{n}\left\langle\mathbf{v}_{j}, \mathbf{e}_{i}\right\rangle \mathbf{e}_{i}$ for $1 \leq j \leq n-1$. Define the $(n-1) \times n$ matrix $R=\left(r_{i j}\right)$ by setting $r_{i j}=\left|\left\langle\mathbf{v}_{i}, \mathbf{e}_{j}\right\rangle\right|^{2}$ for $1 \leq i \leq n-1$ and $1 \leq j \leq n$. Using the fact that $\left(\mathbf{v}_{1}, \ldots, \mathbf{v}_{n-1}, \mathbf{v}_{n}\right)$ is an orthonormal basis of $\mathcal{H}$ one can easily check that

$$
\begin{aligned}
& \sum_{j=1}^{n} r_{i j}=\sum_{j=1}^{n}\left|\left\langle\mathbf{v}_{i}, \mathbf{e}_{j}\right\rangle\right|^{2}=\left\|\mathbf{v}_{i}\right\|^{2}=1, \quad 1 \leq i \leq n-1, \\
& \sum_{i=1}^{n-1} r_{i j}=\sum_{i=1}^{n-1}\left|\left\langle\mathbf{v}_{i}, \mathbf{e}_{j}\right\rangle\right|^{2}=\left\|\mathbf{e}_{j}\right\|^{2}-\left|\left\langle\mathbf{e}_{j}, \mathbf{v}_{n}\right\rangle\right|^{2}=1-a_{j}, \quad 1 \leq j \leq n,
\end{aligned}
$$

so that $R \in \mathbb{M}_{n-1, n}^{r s}$ and $\mathbf{b}=\mathbf{a} R$. Note that by (4.2) one has

$$
\left(w_{1}, \ldots, w_{n-1}\right)^{T}=R\left(z_{1}, \ldots, z_{n}\right)^{T} .
$$

Hence $(W(1,0), \mathbf{a}) \prec(Z(1,0), \mathbf{b})$, which completes the proof of the theorem in the case $\lambda=1$ and $\mu=0$. The general case follows from this one by composing $f$ with a non-singular affine transformation of the plane.

Remark 16 . The compression of a normal operator to a hyperplane is not necessarily normal. For instance, Fan and Pall [15] showed that principal submatrices of a given irreducible normal matrix $B$ are normal if and only if $B$ has collinear eigenvalues.

Remark 17. The proof of Theorem 2 given above is a slight modification of the corresponding arguments in [26] and [28.

We next prove Theorem 3. Clearly, conditions (ii), (iii) and (iv) in this theorem are equivalent by Definition 1 and Theorem 1 Let us first show that (v) $\Rightarrow(\mathrm{i})$. Set

$$
q(z)=\prod_{j=1}^{n-1}\left(z-w_{j}\right), \quad p_{i}(z)=\prod_{\substack{j=1 \\ j \neq i}}^{n}\left(z-z_{j}\right), \quad 1 \leq i \leq n,
$$

and note that condition (i) is equivalent to

$$
q^{(n-1-k)}(0)=\sum_{i=1}^{n} a_{i} p_{i}^{(n-1-k)}(0), \quad 1 \leq k \leq n-1 .
$$

Elementary computations show that

$$
\begin{aligned}
& q^{(n-1-k)}(0)=(-1)^{k}(n-1-k) ! \sum_{1 \leq r_{1}<\ldots<r_{k} \leq n-1} \prod_{j=1}^{k} w_{r_{j}}, \\
& \sum_{i=1}^{n} a_{i} p_{i}^{(n-1-k)}(0)=(-1)^{k}(n-1-k) ! \sum_{1 \leq s_{1}<\ldots<s_{k} \leq n}\left(1-\sum_{i=1}^{k} a_{s_{i}}\right) \prod_{i=1}^{k} z_{s_{i}},
\end{aligned}
$$

for $1 \leq k \leq n-1$. Thus condition (i) amounts to saying that if $1 \leq k \leq n-1$, then

$$
\sum_{1 \leq r_{1}<\ldots<r_{k} \leq n-1} \prod_{j=1}^{k} w_{r_{j}}=\sum_{1 \leq s_{1}<\ldots<s_{k} \leq n}\left(1-\sum_{i=1}^{k} a_{s_{i}}\right) \prod_{i=1}^{k} z_{s_{i}} .
$$


The fact that $(\mathrm{v}) \Rightarrow$ (i) is now a consequence of the following lemma.

Lemma 4. If condition ( $v$ ) in Theorem 3 holds, then (4.3) is true for $1 \leq k \leq n-1$.

Proof. Using, e.g., condition (iii) in Theorem 3 with $\lambda=1$ and $\mu=0$ one gets

$$
\mathbf{a}^{[k]} W_{k}^{[k]}(1,0)^{T}=\mathbf{b}^{[k]} Z_{k}^{[k]}(1,0)^{T}, \quad 1 \leq k \leq n-1,
$$

which is the same as the desired conclusion.

We shall also need the following extension to normal matrices of a well-known result of Schur for Hermitian matrices (cf. [1, 27); see, e.g., 26, Proposition 3.9 (a)].

Lemma 5. If $T=\left(t_{i j}\right)$ is a normal $n \times n$ matrix with eigenvalues $\tau_{1}, \ldots, \tau_{n}$, then

$$
\left(t_{11}, \ldots, t_{n n}\right) \prec\left(\tau_{1}, \ldots, \tau_{n}\right),
$$

i.e., $\left(t_{11}, \ldots, t_{n n}\right)^{T}=S\left(\tau_{1}, \ldots, \tau_{n}\right)^{T}$ for some doubly stochastic $n \times n$ matrix $S$.

Proof. Let $U$ be a unitary matrix such that $T=U \operatorname{diag}\left(\tau_{1}, \ldots, \tau_{n}\right) U^{*}$ and set $S=$ $U \circ \bar{U}$, where $\circ$ denotes the Hadamard-Schur (entrywise) product of matrices and $\bar{U}$ is the complex conjugate of $U$. Then $\left(t_{11}, \ldots, t_{n n}\right)^{T}=S\left(\tau_{1}, \ldots, \tau_{n}\right)^{T}$. It remains to note that $S$ is a unitarily stochastic, hence a doubly stochastic, matrix.

To complete the proof of Theorem 3 it will be convenient to work with certain suitably chosen matrix representations of the operators $A$ and $A^{\prime}$ defined above. As in the proof of Theorem 2 let us fix an orthonormal basis $\left(\mathbf{v}_{1}, \ldots, \mathbf{v}_{n-1}\right)$ of $\mathcal{K}$ in which the matrix representation of the $P$-compression $A^{\prime}$ of $A$ is upper triangular. For simplicity, denote the matrix representation of $A$ in the basis $\left(\mathbf{v}_{1}, \ldots, \mathbf{v}_{n-1}, \mathbf{v}_{n}\right)$ again by $A$ and let $A_{i}, 1 \leq i \leq n$, be the degeneracy one principal submatrix of $A$ obtained by deleting its $i$-th row and $i$-th column. Then clearly $A_{n}=A^{\prime}$, and so by Lemma 3 the spectrum of $A_{n}$ is $\Sigma\left(A_{n}\right)=\left\{w_{1}, \ldots, w_{n-1}\right\}$, where $\Sigma\left(A_{n}\right)$ is viewed as a multiset whose elements occur as many times as their algebraic multiplicities as eigenvalues of $A_{n}$. Thus $A$ is an $n \times n$ normal matrix with $\Sigma(A)=\left\{z_{1}, \ldots, z_{n}\right\}$ such that

$$
A=\left(\begin{array}{cc}
A_{n} & * \\
* & \zeta
\end{array}\right)
$$

where $A_{n}$ is upper triangular with $\Sigma\left(A_{n}\right)=\left\{w_{1}, \ldots, w_{n-1}\right\}$ and $\zeta=\left\langle A \mathbf{v}_{n}, \mathbf{v}_{n}\right\rangle=$ $\sum_{i=1}^{n} a_{i} z_{i}$ is the $\left(a_{1}, \ldots, a_{n}\right)$-barycenter of $Z(1,0)$ (cf. Remark 8$)$. Note that since the $w_{j}$ appear on the diagonal of $A_{n}$ Lemma 5 actually provides a new proof of Theorem 2, Let $U=\left(u_{i j}\right)$ be the (unitary) change of basis matrix from $\left(\mathbf{v}_{1}, \ldots, \mathbf{v}_{n}\right)$ to $\left(\mathbf{e}_{1}, \ldots, \mathbf{e}_{n}\right)$ so that we may write

$$
A=U \operatorname{diag}\left(z_{1}, \ldots, z_{n}\right) U^{*} .
$$

Proof of Theorem 13. Let us first show that if (3.6) holds and $k \in\{1, \ldots, n-1\}$, then one can find an $\left(\begin{array}{c}n-1 \\ k\end{array}\right) \times\left(\begin{array}{l}n \\ k\end{array}\right)$ row stochastic matrix $R_{k}$ such that

$$
W_{k}^{[k]}(1,0)^{T}=R_{k} Z_{k}^{[k]}(1,0)^{T} \text { and } \mathbf{b}^{[k]}=\mathbf{a}^{[k]} R_{k} .
$$

This is clear for $k=1$ by Theorem 2 and so we may assume that $k \geq 2$. Let

$$
A^{(k)}=(\operatorname{det} A(\mathbf{i}, \mathbf{j}))_{\mathbf{i} . \mathbf{j} \in Q_{k, n}}
$$

be the $k$-th compound matrix of $A$ acting on the $k$-th Grassmann power $\wedge^{k} \mathcal{H}$ (recall that the elements of $Q_{k, n}$ and therefore also the $\left(\begin{array}{l}n \\ k\end{array}\right) \times\left(\begin{array}{l}n \\ k\end{array}\right)$ determinants 
appearing in $A^{(k)}$ are arranged in lexicographic order). The Binet-Cauchy formula for compound matrices [27, Theorem 19.F.2] and (4.5) imply that

$$
A^{(k)}=U^{(k)} \operatorname{diag}\left(z_{1}, \ldots, z_{n}\right)^{(k)} U^{*(k)},
$$

which by an argument similar to the proof of Lemma 5 leads to

$$
(\operatorname{det} A(\mathbf{i}, \mathbf{i}))_{\mathbf{i} \in Q_{k, n}}^{T}=S_{k} Z_{k}^{[k]}(1,0)^{T}, \quad S_{k}=U^{(k)} \circ \bar{U}^{(k)},
$$

where o denotes as before the Hadamard-Schur product. Note that $S_{k}$ is an $\left(\begin{array}{l}n \\ k\end{array}\right) \times\left(\begin{array}{l}n \\ k\end{array}\right)$ doubly stochastic matrix since $U^{(k)}$ is unitary. Clearly, the diagonal elements of $A^{(k)}$ corresponding to the $k \times k$ principal minors of $A_{n}$ are of the form $\operatorname{det} A(\mathbf{i}, \mathbf{i})$, $\mathbf{i} \in Q_{k, n-1}$. There are a total of $\left(\begin{array}{c}n-1 \\ k\end{array}\right)$ such diagonal elements, and these must actually coincide with the coordinates of the vector $W_{k}^{[k]}(1,0)^{T}$ since $A_{n}$ is upper triangular. Let $R_{k}$ be the $\left(\begin{array}{c}n-1 \\ k\end{array}\right) \times\left(\begin{array}{c}n \\ k\end{array}\right)$ row stochastic matrix obtained from $S_{k}$ by deleting all elements $S_{k}(\mathbf{i}, \mathbf{j})$ with $\mathbf{i} \in Q_{k, n} \backslash Q_{k, n-1}$. We show that $R_{k}$ satisfies (4.6). For this one has to prove that for any $\mathbf{j}=\left(j_{1}, \ldots, j_{k}\right) \in Q_{k, n}$ the identity

$$
\sum_{1 \leq i_{1}<\ldots<i_{k-1} \leq n-1} S_{k}\left(\begin{array}{c}
i_{1}, \ldots, i_{k-1}, n \\
j_{1}, \ldots, j_{k}
\end{array}\right)=\sum_{l=1}^{k} a_{j_{l}}
$$

holds, which is equivalent to

$$
\sum_{1 \leq i_{1}<\ldots<i_{k-1} \leq n-1}\left|\operatorname{det} U\left(\begin{array}{c}
i_{1}, \ldots, i_{k-1}, n \\
j_{1}, \ldots, j_{k}
\end{array}\right)\right|^{2}=\sum_{l=1}^{k} a_{j_{l}}
$$

or

$$
\sum_{\mathbf{i} \in Q_{k, n-1}}|\operatorname{det} U(\mathbf{i}, \mathbf{j})|^{2}=1-\sum_{l=1}^{k} a_{j_{l}} .
$$

By (4.5) one has $u_{n j}=\sqrt{a_{j}}, 1 \leq j \leq n$, so that by expanding each determinant with respect to the last row we get

$$
\operatorname{det} U\left(\begin{array}{c}
i_{1}, \ldots, i_{k-1}, n \\
j_{1}, \ldots, j_{k}
\end{array}\right)=\sum_{m=1}^{k}(-1)^{k+m} \sqrt{a_{j_{m}}} \operatorname{det} U\left(\begin{array}{c}
i_{1}, \ldots, i_{k-1} \\
j_{1}, \ldots, \widehat{j_{m}}, \ldots, j_{k}
\end{array}\right)
$$

and thus

$$
\begin{aligned}
& \left|\operatorname{det} U\left(\begin{array}{c}
i_{1}, \ldots, i_{k-1}, n \\
j_{1}, \ldots, j_{k}
\end{array}\right)\right|^{2} \\
& \quad=\sum_{r, s=1}^{k}(-1)^{r+s} \sqrt{a_{j_{r}} a_{j_{s}}} \operatorname{det} U\left(\begin{array}{c}
i_{1}, \ldots, i_{k-1} \\
j_{1}, \ldots, \hat{j_{r}}, \ldots, j_{k}
\end{array}\right) \operatorname{det} \bar{U}\left(\begin{array}{c}
i_{1}, \ldots, i_{k-1} \\
j_{1}, \ldots, \widehat{j_{s}}, \ldots, j_{k}
\end{array}\right)
\end{aligned}
$$

for $1 \leq i_{1}<\ldots<i_{k-1} \leq n-1$. Therefore

$$
\sum_{1 \leq i_{1}<\ldots<i_{k-1} \leq n-1}\left|\operatorname{det} U\left(\begin{array}{c}
i_{1}, \ldots, i_{k-1}, n \\
j_{1}, \ldots, j_{k}
\end{array}\right)\right|^{2}=\sum_{r, s=1}^{k}(-1)^{r+s} \sqrt{a_{j_{r}} a_{j_{s}}} \alpha_{r, s}(\mathbf{j}),
$$

where

$$
\alpha_{r, s}(\mathbf{j})=\sum_{1 \leq i_{1}<\ldots<i_{k-1} \leq n-1} \operatorname{det} U\left(\begin{array}{c}
i_{1}, \ldots, i_{k-1} \\
j_{1}, \ldots, \hat{j_{r}}, \ldots, j_{k}
\end{array}\right) \operatorname{det} \bar{U}\left(\begin{array}{c}
i_{1}, \ldots, i_{k-1} \\
j_{1}, \ldots, \hat{j_{s}}, \ldots, j_{k}
\end{array}\right) .
$$


Let us consider the $k \times(n-1)$ matrix

$$
M=U\left(\begin{array}{c}
1, \ldots, n-1 \\
j_{1}, \ldots, j_{k}
\end{array}\right)^{T}
$$

and the $k \times k$ matrix $B=\left(b_{i j}\right)=M M^{*}$ as well as the $k \times k$ matrix given by its $k-1$ Grassmann power $B^{(k-1)}=\left(b_{i j}^{(k-1)}\right)$. The Binet-Cauchy formula again implies that

$$
B^{(k-1)}=M^{(k-1)} M^{*(k-1)}
$$

from which we deduce that

$$
b_{k+1-r, k+1-s}^{(k-1)}=\alpha_{r, s}(\mathbf{j}), \quad 1 \leq r, s \leq k .
$$

Thus the left-hand side of (4.8) equals

$$
\sum_{r, s=1}^{k}(-1)^{r+s} \sqrt{a_{j_{r}} a_{j_{s}}} b_{k+1-r, k+1-s}^{(k-1)}
$$

and by further manipulating this expression we obtain

$$
\sum_{1 \leq i_{1}<\ldots<i_{k-1} \leq n-1}\left|\operatorname{det} U\left(\begin{array}{c}
i_{1}, \ldots, i_{k-1}, n \\
j_{1}, \ldots, j_{k}
\end{array}\right)\right|^{2}=\sum_{\mathbf{i} \in Q_{k-1, k}} \operatorname{det} B(\mathbf{i}, \mathbf{i})-k \operatorname{det} B
$$

hence

$$
\sum_{\mathbf{i} \in Q_{k, n-1}}|\operatorname{det} U(\mathbf{i}, \mathbf{j})|^{2}=1-\sum_{\mathbf{i} \in Q_{k-1, k}} \operatorname{det} B(\mathbf{i}, \mathbf{i})+k \operatorname{det} B .
$$

To show that the right-hand side of the above identity equals the right-hand side of (4.9) we proceed as follows. Define the vector $\mathbf{u}=\left(\sqrt{a_{j_{1}}}, \ldots, \sqrt{a_{j_{k}}}\right)$. Since $U$ is unitary an easy computation shows that $B=I_{k}-\mathbf{u}^{T} \mathbf{u}$, so that $B$ depends only on the numbers $a_{j_{l}}, 1 \leq l \leq k$. It follows that the expression

$$
\sum_{1 \leq i_{1}<\ldots<i_{k} \leq n-1}\left|\operatorname{det} U\left(\begin{array}{c}
i_{1}, \ldots, i_{k} \\
j_{1}, \ldots, j_{k}
\end{array}\right)\right|^{2}=\sum_{\mathbf{i} \in Q_{k, n-1}}|\operatorname{det} U(\mathbf{i}, \mathbf{j})|^{2}
$$

also depends exclusively on the numbers $a_{j_{l}}, 1 \leq l \leq k$, and is therefore independent of the $z_{i}, 1 \leq i \leq n$. On the other hand, by summing all coordinates corresponding to $\mathbf{i} \in Q_{k, n-1}$ in each side of (4.7) we get

$$
\begin{aligned}
& \sum_{1 \leq r_{1}<\ldots<r_{k} \leq n-1} \prod_{j=1}^{k} w_{r_{j}} \\
= & \sum_{1 \leq s_{1}<\ldots<s_{k} \leq n}\left(\sum_{1 \leq i_{1}<\ldots<i_{k} \leq n-1}\left|\operatorname{det} U\left(\begin{array}{c}
i_{1}, \ldots, i_{k} \\
s_{1}, \ldots, s_{k}
\end{array}\right)\right|^{2}\right) \prod_{i=1}^{k} z_{s_{l}} .
\end{aligned}
$$

Obviously, (3.6) defines the $w_{j}, 1 \leq j \leq n-1$, as continuous functions of the $z_{i}, 1 \leq i \leq n$. By letting $z_{i} \rightarrow 0$ for $i \in\{1, \ldots, n\} \backslash\left\{j_{1}, \ldots, j_{k}\right\}$ and $z_{j_{l}} \rightarrow 1$ for $1 \leq l \leq k$ in both (4.10) and (4.3) and comparing coefficients in the resulting identities we get (4.9), which proves (4.6).

Finally, to prove the last statement of the theorem it is enough to consider the function $f\left(\lambda^{-1}(z-\mu)\right)$, where $\lambda \in \mathbb{C} \backslash\{0\}$ and $\mu \in \mathbb{C}$. The zeros and poles of this function are the eigenvalues of $\lambda A_{n}+\mu$ and $\lambda A+\mu$, respectively. These matrices 
satisfy relations similar to (4.4) and (4.5), while the unitary matrix $U$ is unchanged. One may therefore use the same arguments as above to get (3.7).

Remark 18. Modulo some technical modifications that have to deal with unequal weights, the arguments given in the proof of Theorem 3 are the same as those given by S. Malamud in his proof of [26, Theorem 4.7].

Remark 19. An interesting byproduct of the proof of Theorem 3 is the following identity for certain types of $M$-matrices. Let $m \in \mathbb{N}, \mathbf{x}=\left(x_{1}, \ldots, x_{m}\right) \in(0,1)^{m}$ with $\|\mathbf{x}\|^{2}=\sum_{i=1}^{m} x_{i}^{2}<1$ and define the $m \times m$ matrix $X=I_{m}-\mathbf{x}^{T} \mathbf{x}$. Clearly, $\mathbf{x}^{T} \mathbf{x}$ is a rank one matrix whose eigenvalues 0 (with multiplicity $m-1$ ) and $\|\mathbf{x}\|^{2}$ have modulus less than 1 , so that $X$ is an $M$-matrix (see, e.g., 27]). By the arguments in the last part of the proof of Theorem 3 we see that

$$
\sum_{\mathbf{i} \in Q_{m-1, m}} \operatorname{det} X(\mathbf{i}, \mathbf{i})-m \operatorname{det} X=\operatorname{tr}\left(I_{m}-X\right),
$$

both sides being equal to $\|\mathbf{x}\|^{2}$.

Recall that if $C$ is an $n \times n$ matrix and $1 \leq k \leq n$ one defines the $k$-th additive compound matrix $\Delta_{k}(C)$ as the $\left(\begin{array}{l}n \\ k\end{array}\right) \times\left(\begin{array}{l}n \\ k\end{array}\right)$ given by

$$
\Delta_{k}(C)=\left.\frac{d}{d t}\left(I_{n}+t C\right)^{(k)}\right|_{t=0} .
$$

Equivalently, $\Delta_{k}(C)$ is the coefficient matrix of $t$ in the expansion $\left(I_{n}+t C\right)^{(k)}=$ $I_{n}+t \Delta_{k}(C)+\mathcal{O}\left(t^{2}\right)$. As an operator, this is the same as the restriction to $\bigwedge^{k} \mathcal{H}$ of the operator

$$
C \otimes I_{n} \otimes \cdots \otimes I_{n}+I_{n} \otimes C \otimes I_{n} \otimes \cdots \otimes I_{n}+\ldots+I_{n} \otimes I_{n} \otimes \cdots \otimes C
$$

defined on $\otimes^{k} \mathcal{H}$. It is well known that if $C$ has eigenvalues $\lambda_{1}, \ldots, \lambda_{n}$, then the eigenvalues of $\Delta_{k}(C)$ are $\lambda_{i_{1}}+\ldots+\lambda_{i_{k}}, 1 \leq i_{1}<\ldots<i_{k} \leq n$ (cf., e.g., [27]).

Proof of Corollary 4, Using (4.5) and arguing as in the first part of the proof of Theorem 3 we arrive at

$$
\left.\frac{d}{d t}\left(I_{n}+t A\right)^{(k)}\right|_{t=0}=\left.U^{(k)} \frac{d}{d t}\left(\operatorname{diag}\left(1+t z_{1}, \ldots, 1+t z_{n}\right)^{(k)}\right)\right|_{t=0} U^{*(k)} .
$$

Since $A_{n}$ is upper triangular the diagonal elements of $\left(I_{n}+t A\right)^{(k)}$ corresponding to the $k \times k$ principal minors indexed by multi-indices $\mathbf{i}=\left(i_{1}, \ldots, i_{k}\right) \in Q_{k, n-1}$ have the form $\prod_{j=1}^{k}\left(1+t w_{i_{j}}\right)$. The conclusion now follows from the obvious identities

$$
\left.\frac{d}{d t} \prod_{j=1}^{k}\left(1+t w_{i_{j}}\right)\right|_{t=0}=\sum_{j=1}^{k} w_{i_{j}}, \quad \frac{d}{d t} \prod_{l=1}^{k}\left(1+t z_{s_{l}}\right)=\sum_{l=1}^{k} z_{s_{l}}
$$

combined with the arguments in the second part of the proof of Theorem 3 .

Corollary 4 may alternatively be proved by using (3.7) with $\lambda=t$ and $\mu=1$ and differentiating with respect to $t$. Using this alternative method we can actually prove Corollary 3 .

Proof of Corollary 3, Let $\lambda \in \mathbb{C} \backslash\{0\}$ and $\mu \in \mathbb{C}$. By (3.7) and Remark 11 there exists an $\left(\begin{array}{c}n-1 \\ k\end{array}\right) \times\left(\begin{array}{l}n \\ k\end{array}\right)$ row stochastic matrix $R_{k}$ such that the relations

$$
W_{k}^{[k]}(t \lambda, t \mu+1)^{T}=R_{k} Z_{k}^{[k]}(t \lambda, t \mu+1)^{T} \text { and } \mathbf{b}^{[k]}=\mathbf{a}^{[k]} R_{k}
$$


hold for all $t \in \mathbb{C}$. Thus

$$
\left.\frac{d^{m}}{d t^{m}} W_{k}^{[k]}(t \lambda, t \mu+1)^{T}\right|_{t=0}=\left.R_{k} \frac{d^{m}}{d t^{m}} Z_{k}^{[k]}(t \lambda, t \mu+1)^{T}\right|_{t=0}
$$

for $m \in\{1, \ldots, k\}$, which is the same as (3.8).

Remark 20. The assumption that the points $z_{i}, 1 \leq i \leq n$, are distinct that was made at the beginning of $\$ 3.1$ was not actually used in the above proofs. However, this condition is natural when viewing - as we did - these points as charged particles generating a resultant electrostatic force given by (3.2). Clearly, the latter can be appropriately rewritten even in the case of multiple points.

4.2. Proof of Theorems 45 . The inequality $h(\mathcal{W}, \mathcal{Z}) \leq h\left(\mathcal{W}_{e}, \mathcal{Z}\right)$ is trivially true since $\mathcal{W} \subseteq \mathcal{W}_{e}$. To prove the interesting part of Theorem 4 we make use of a result on spectral variations of normal matrices which is usually referred to as the Bauer-Fike theorem (cf., e.g., [1, Theorem VI.3.3]).

Lemma 6. Let $A$ and $B$ be $n \times n$ complex matrices. If $A$ is normal, then

$$
h(\Sigma(B), \Sigma(A)) \leq\|A-B\|,
$$

where $h$ denotes the relative Hausdorff distance from $\Sigma(B)$ to $\Sigma(A)$.

Recall the orthonormal basis $\left(\mathbf{v}_{1}, \ldots, \mathbf{v}_{n}\right)$ of $\mathcal{H}$ where the matrix representation of the normal operator $A$ with $\Sigma(A)=\mathcal{Z}$ is given by (4.4), that is,

$$
A=\left(\begin{array}{cc}
A_{n} & \mathbf{x} \\
\mathbf{y}^{*} & \zeta
\end{array}\right)
$$

In the above decomposition $A_{n}$ is an $(n-1) \times(n-1)$ upper triangular matrix with $\Sigma\left(A_{n}\right)=\mathcal{W}, \zeta=\left\langle A \mathbf{v}_{n}, \mathbf{v}_{n}\right\rangle=\sum_{i=1}^{n} a_{i} z_{i}$, and $\mathbf{x}, \mathbf{y} \in \mathbb{C}^{n-1}$. Set $B=A_{n} \oplus \zeta$. An easy computation now shows that

$$
\begin{aligned}
\|A-B\|^{2} & =\|\mathbf{x}\|^{2}=\|\mathbf{y}\|^{2}=\sum_{i=1}^{n-1}\left|\left\langle A \mathbf{v}_{n}, \mathbf{v}_{i}\right\rangle\right|^{2}=\left\|A \mathbf{v}_{n}\right\|^{2}-\left|\left\langle A \mathbf{v}_{n}, \mathbf{v}_{n}\right\rangle\right|^{2} \\
& =\sum_{i=1}^{n} a_{i}\left|z_{i}\right|^{2}-|\zeta|^{2}=\sigma_{2}(\mathbf{z} ; \mathbf{a})^{2}
\end{aligned}
$$

which combined with Lemma 6 yields the inequality stated in Theorem 4.

To prove Theorem 5 note first that by Theorem 4 it remains to check that $h\left(\mathcal{Z}, \mathcal{W}_{e}\right) \leq \sigma_{2}(\mathbf{z} ; \mathbf{a})$. The latter inequality is invariant under nonsingular affine transformations of the complex plane, and it is therefore enough to show that $h\left(\mathcal{Z}, \mathcal{W}_{e}\right) \leq \sigma_{2}(\mathbf{z} ; \mathbf{a})$ whenever $\mathbf{z}=\left(z_{1}, \ldots, z_{n}\right) \in \mathbb{R}^{n}$. Since in this case $A$ is a Hermitian operator we get from (4.11) that $\mathbf{x}:=\left(x_{1}, \ldots, x_{n-1}\right)^{t}=\mathbf{y}$ and $A_{n}=$ $\operatorname{diag}\left(w_{1}, \ldots, w_{n-1}\right)$. Suppose $z_{i}$ is such that

$$
\min _{1 \leq j \leq n-1}\left|z_{i}-w_{j}\right|>\sigma_{2}(\mathbf{z} ; \mathbf{a})
$$

and let $\mathbf{u}=\left(u_{1}, \ldots, u_{n}\right)^{t} \in \mathbb{C}^{n}$ be a unit eigenvector of $A$ with eigenvalue $z_{i}$. Then by (4.11) we may rewrite the identity $A \mathbf{u}=z_{i} \mathbf{u}$ as

$$
\left(\zeta-z_{i}\right) u_{n}=-\sum_{j=1}^{n-1} \bar{x}_{j} u_{j} \text { and }\left(w_{j}-z_{i}\right) u_{j}=x_{j} u_{n}, \quad 1 \leq j \leq n-1 .
$$


Note that $\sigma_{2}(\mathbf{z} ; \mathbf{a})>0$ since $z_{1}, \ldots, z_{n}$ are assumed to be pairwise distinct. Moreover, the fact that $\|\mathbf{u}\|=1$ combined with (4.13) implies that $u_{n} \neq 0$. From (4.12) and the last $n-1$ identities in (4.13) we get

$$
\left|u_{j}\right| \leq \sigma_{2}(\mathbf{z} ; \mathbf{a})^{-1}\left|x_{j}\right|\left|u_{n}\right|, \quad 1 \leq j \leq n-1,
$$

and then using the first relation in (4.13) we deduce that

$$
\left|\zeta-z_{i}\right| \leq \sigma_{2}(\mathbf{z} ; \mathbf{a})^{-1}\|\mathbf{x}\|^{2}=\sigma_{2}(\mathbf{z} ; \mathbf{a}) .
$$

This shows that $h\left(\left\{z_{i}\right\}, \mathcal{W}_{e}\right) \leq \sigma_{2}(\mathbf{z} ; \mathbf{a})$, which completes the proof of Theorem 5 ,

4.3. Proof of Theorems 6-7. It is clear that up to a rescaling we may assume that $\rho=1$ in both Theorem 6 and Theorem 7. Below we shall use the Hilbert space of square summable complex sequences $\mathcal{H}=l^{2}(\mathbb{N})$ with standard scalar product $\langle\cdot, \cdot\rangle$ and standard orthonormal basis $\left\{\mathbf{e}_{i}\right\}_{i \in \mathbb{N}}$.

Proof of Theorem [6. We may assume wlog that $\left\{z_{i}\right\}_{i \in \mathbb{N}}$ is an increasing sequence. The fact that $f$ has infinitely many zeros $\left\{w_{j}\right\}_{j \in \mathbb{N}}$ that interlace the poles $\left\{z_{i}\right\}_{i \in \mathbb{N}}$ on the real axis follows by noticing that all zeros of $f$ are necessarily real and that $f$ is strictly decreasing on each interval $\left(z_{i}, z_{i+1}\right), i \in \mathbb{N}$. Alternatively, this may also be viewed as a consequence of the operator theoretic interpretation given below combined with Hochstadt's interlacing theorem for the eigenvalues of rank one perturbations of compact selfadjoint Hilbert space operators [20].

Let $A$ denote the unique bounded operator on $\mathcal{H}$ satisfying $A \mathbf{e}_{i}=\left(1-z_{i}\right) \mathbf{e}_{i}$, $i \in \mathbb{N}$. Note that $A$ is injective, selfadjoint and compact since the $z_{i}$ are real and $z_{i} \rightarrow 1$ as $i \rightarrow \infty$. By assumption one has $\sum_{i=1}^{\infty} a_{i}=1$, and so we may define a unit vector $\mathbf{v} \in \mathcal{H}$ in the following way:

$$
\mathbf{v}=\sum_{i=1}^{\infty} \sqrt{a_{i}} \mathbf{e}_{i}
$$

Set $\mathcal{K}=\mathbf{v}^{\perp}$ and let $P$ be the orthoprojection on $\mathcal{K}$. Recall from [22, $\left.\S I V .6\right]$ that the Weinstein-Aronszajn determinant of the second kind associated with $A$ and $P$ is given by $\left\langle(A-z I)^{-1} \mathbf{v}, \mathbf{v}\right\rangle$. A simple computation shows that

$$
\left\langle(A-z I)^{-1} \mathbf{v}, \mathbf{v}\right\rangle=f(1-z), \quad z \in \mathbb{C} \backslash\left\{1-z_{i}\right\}_{i \in \mathbb{N}} .
$$

Let us now consider the compression $A^{\prime}$ of $A$ to $\mathcal{K}$, that is, the operator on $\mathcal{K}$ given by $A^{\prime}=\left.P A P\right|_{\mathcal{K}}$. Note that $A^{\prime}$ is itself a compact selfadjoint operator. We claim that the point spectrum of $A^{\prime}$ consists precisely of the zeros of $f(1-z)$.

Lemma 7. The set of eigenvalues of $A^{\prime}$ is $\left\{1-w_{j}\right\}_{j \in \mathbb{N}}$.

Proof. If $\mathbf{u} \in \mathcal{K}$ is an eigenvector of $A^{\prime}$ with eigenvalue $\zeta \in \mathbb{C}$ (actually $\mathbb{R}$ ), then

$$
(A-\zeta I) \mathbf{u}=(I-P) A \mathbf{u}+\left(A^{\prime}-\zeta I\right) \mathbf{u}=\langle A \mathbf{u}, \mathbf{v}\rangle \mathbf{v} .
$$

From (4.16) we see that $\langle A \mathbf{u}, \mathbf{v}\rangle \neq 0$ since otherwise $\mathbf{u}$ would be an eigenvector of $A$, say $\mathbf{u}=\mathbf{e}_{i}$ for some $i \in \mathbb{N}$, which would then lead to the contradiction $\langle A \mathbf{u}, \mathbf{v}\rangle=\left\langle A \mathbf{e}_{i}, \mathbf{v}\right\rangle=\left(1-z_{i}\right) \sqrt{a_{i}} \neq 0$. By (4.15) and (4.16) we get

$$
f(1-\zeta)=\left\langle(A-\zeta I)^{-1} \mathbf{v}, \mathbf{v}\right\rangle=\langle A \mathbf{u}, \mathbf{v}\rangle^{-1}\langle\mathbf{u}, \mathbf{v}\rangle=0
$$

so that $1-\zeta=w_{j}$ for some $j \in \mathbb{N}$. Conversely, let $w$ be such that $f(1-w)=0$, that is, $\left\langle(A-w I)^{-1} \mathbf{v}, \mathbf{v}\right\rangle=0$, and set $\mathbf{u}=(A-w I)^{-1} \mathbf{v}$. Then clearly $\mathbf{u} \neq 0$ and one has

$$
\mathbf{v}=(A-w I) \mathbf{u}=\langle A \mathbf{u}, \mathbf{v}\rangle \mathbf{v}+\left(A^{\prime}-w I\right) \mathbf{u}
$$


Since $\left(A^{\prime}-w I\right) \mathbf{u} \in \mathcal{K}$ and $\mathbf{v} \in \mathcal{K}^{\perp}$ it follows that

$$
\left(A^{\prime}-w I\right) \mathbf{u}=(1-\langle A \mathbf{u}, \mathbf{v}\rangle) \mathbf{v}=0 .
$$

Thus $\mathbf{u}$ is an eigenvector of $A^{\prime}$ with eigenvalue $w$.

It is not difficult to see that $A^{\prime}$ is injective. Indeed, if $0 \neq \mathbf{u} \in \mathcal{K}$ is such that $A^{\prime} \mathbf{u}=0$, then from $P A \mathbf{u}=0$ and the fact that $A$ is injective we get $A \mathbf{u}=\alpha \mathbf{v}$ for some $\alpha \in \mathbb{C} \backslash\{0\}$. Hence

$$
0=\langle\mathbf{u}, \mathbf{v}\rangle=\alpha\left\langle A^{-1} \mathbf{v}, \mathbf{v}\right\rangle=\alpha f(1),
$$

which is a contradiction since the assumptions of the theorem (more specifically, condition (3.14) with $\rho=1$ ) imply that $f(1)>0$.

By the spectral theorem for compact selfadjoint operators [11, Corollary X.3.3.5] there exists a complete orthonormal system $\left\{\mathbf{f}_{j}\right\}_{j \in \mathbb{N}}$ in $\mathcal{K}$ consisting of eigenvectors for $A^{\prime}$. Set $s_{j k}=\left|\left\langle\mathbf{f}_{j}, \mathbf{e}_{k}\right\rangle\right|^{2}$ for $j, k \in \mathbb{N}$. Then

$$
1-w_{j}=\left\langle A^{\prime} \mathbf{f}_{j}, \mathbf{f}_{j}\right\rangle=\left\langle A \mathbf{f}_{j}, \mathbf{f}_{j}\right\rangle=\sum_{k=1}^{\infty} s_{j k}\left(1-z_{k}\right) .
$$

Both $\{\mathbf{v}\} \cup\left\{\mathbf{f}_{j}\right\}_{j \in \mathbb{N}}$ and $\left\{\mathbf{e}_{k}\right\}_{j \in \mathbb{N}}$ are complete orthonormal systems in $\mathcal{H}$, so that

$$
\begin{aligned}
& \sum_{k=1}^{\infty} s_{j k}=\sum_{k=1}^{\infty}\left|\left\langle\mathbf{f}_{j}, \mathbf{e}_{k}\right\rangle\right|^{2}=\left\|\mathbf{f}_{j}\right\|^{2}=1, \quad j \in \mathbb{N}, \\
& \sum_{j=1}^{\infty} s_{j k}=\sum_{j=1}^{\infty}\left|\left\langle\mathbf{f}_{j}, \mathbf{e}_{k}\right\rangle\right|^{2}=\left\|\mathbf{e}_{k}\right\|^{2}-\left|\left\langle\mathbf{v}, \mathbf{e}_{k}\right\rangle\right|^{2}=1-a_{k}, \quad k \in \mathbb{N},
\end{aligned}
$$

which completes the proof of the theorem.

Proof of Theorem 17. Let $\left\{z_{i}\right\}_{i \in \mathbb{N}}$ be a sequence of distinct complex numbers satisfying (3.14) with $\rho=1$ and let $A$ be the injective compact normal operator on $\mathcal{H}$ defined by $A \mathbf{e}_{i}=\left(1-z_{i}\right) \mathbf{e}_{i}, i \in \mathbb{N}$. As before we denote by $A^{\prime}$ the compression $\left.P A P\right|_{\mathcal{K}}$ of $A$, where $\mathcal{K}=\mathbf{v}^{\perp}$ and $\mathbf{v}$ is given by (4.14). Clearly, $A^{\prime}$ is a compact operator on $\mathcal{K}$. Moreover, since $\Re f(1)>0$ the arguments in the proof of Theorem 6 show that $A^{\prime}$ is injective. Note that Lemma 7 remains valid in the present setting and that by assumption $f$ has a discrete set of zeros $\left\{w_{j}\right\}_{j \in \mathbb{N}}$. It follows that the point spectrum of $A^{\prime}$ is $\left\{1-w_{j}\right\}_{j \in \mathbb{N}}$. Denote by $\mathcal{K}_{A^{\prime}}$ the closed linear hull of the root subspaces of $A^{\prime}$ and let $\widehat{A^{\prime}}$ be the operator on $\mathcal{K}_{A^{\prime}}$ induced by $A^{\prime}$. Recall the operator version of Schur's lemma given in [17, Lemma 4.1].

Lemma 8. With the above notations there exists an orthonormal basis $\left\{\mathbf{f}_{j}\right\}_{j \in \mathbb{N}}$ of $\mathcal{K}_{A^{\prime}}$ in which the matrix of the operator $\widehat{A^{\prime}}$ has triangular form and $\left\langle\widehat{A}^{\prime} \mathbf{f}_{j}, \mathbf{f}_{j}\right\rangle=$ $1-w_{j}, j \in \mathbb{N}$.

Set $s_{j k}=\left|\left\langle\mathbf{f}_{j}, \mathbf{e}_{k}\right\rangle\right|^{2}$ for $j, k \in \mathbb{N}$. By Lemma 8 we have

$$
1-w_{j}=\left\langle\widehat{A^{\prime}} \mathbf{f}_{j}, \mathbf{f}_{j}\right\rangle=\left\langle A \mathbf{f}_{j}, \mathbf{f}_{j}\right\rangle=\sum_{k=1}^{\infty} s_{j k}\left(1-z_{k}\right) .
$$

It is clear that

$$
\sum_{k=1}^{\infty} s_{j k}=\sum_{k=1}^{\infty}\left|\left\langle\mathbf{f}_{j}, \mathbf{e}_{k}\right\rangle\right|^{2}=\left\|\mathbf{f}_{j}\right\|^{2}=1, \quad j \in \mathbb{N} .
$$


On the other hand $\{\mathbf{v}\} \cup\left\{\mathbf{f}_{j}\right\}_{j \in \mathbb{N}}$ is an orthonormal system in $\mathcal{H}$ so that by Bessel's inequality we get

$$
\sum_{j=1}^{\infty} s_{j k}=\sum_{j=1}^{\infty}\left|\left\langle\mathbf{f}_{j}, \mathbf{e}_{k}\right\rangle\right|^{2} \leq\left\|\mathbf{e}_{k}\right\|^{2}-\left|\left\langle\mathbf{v}, \mathbf{e}_{k}\right\rangle\right|^{2}=1-a_{k}, \quad k \in \mathbb{N} .
$$

The inequality stated in the theorem by means of nonnegative convex functions is an immediate consequence of (4.17)-(4.18).

\section{A hierarchy of De BruiJn-Springer RElations and inertia laWs}

Theorems 2 3 suggest even deeper physical connections between the set of equilibrium points and the given set of charged particles in the case of finite planar systems, such as a whole new hierarchy of inertia laws for planar solid bodies associated with these sets. In order to make a precise statement let us identify the complex plane $\mathbb{C}$ with the $x_{1} x_{2}$-plane in $\mathbb{R}^{3}$ equipped with the standard coordinate system $\left(O ; x_{1}, x_{2}, x_{3}\right)$. For an integer $k \geq 2$ we denote by $\Xi_{k}$ the standard simplex

$$
\Xi_{k}=\left\{\left(t_{1}, \ldots, t_{k}\right) \in[0,1]^{k} \mid t_{1}+\ldots+t_{k}=1\right\} .
$$

Definition 3. Let $L$ be a line in $\mathbb{R}^{3}$. Given a convex $k$-gon $\mathcal{K}$ in $\mathbb{C}$ with vertices $v_{1}, \ldots, v_{k}$ and $\alpha \geq 1$ we define the $\alpha$-moment of $\mathcal{K}$ with respect to $L$ to be

$$
I_{\alpha, L}(\mathcal{K})=\int \cdots \int_{\Xi_{k}} d\left(t_{1} v_{1}+\ldots+t_{k} v_{k}, L\right)^{\alpha} d t_{1} \cdots d t_{k},
$$

where $d(\cdot, L)$ denotes the distance function associated with $L$.

Remark 21. If $\mathcal{K}$ is viewed as a planar solid body with uniform mass distribution, then $I_{\alpha, L}(\mathcal{K})$ may be interpreted as a higher moment of inertia (or generalized angular momentum) of $\mathcal{K}$ with respect to the rotation axis $L$.

Let $\Sigma_{m}$ denote the symmetric group on $m$ elements. It is not difficult to see that if $\mathcal{K}$ is as above and $u_{1}, \ldots, u_{m} \in \mathbb{C}$ are such that $\mathcal{K}=\operatorname{conv}\left(u_{1}, \ldots, u_{m}\right)$, then

$$
\int \cdots \int_{\Xi_{m}} d\left(t_{\pi(1)} u_{1}+\ldots+t_{\pi(m)} u_{m}, L\right)^{\alpha} d t_{1} \cdots d t_{m}=I_{\alpha, L}(\mathcal{K})
$$

for any $\pi \in \Sigma_{m}$, so that $I_{\alpha, L}(\mathcal{K})$ depends only on the extreme points of $\mathcal{K}$.

The following conjecture is a powerful generalization of Theorems 23 and suggests a "hierarchy" of weighted de Bruijn-Springer relations and inertia laws for planar polygons constructed from the equilibrium points and the charged particles.

Conjecture 1. Let $z_{i}, 1 \leq i \leq n$, be distinct points in $\mathbb{C}$ and $a_{i}>0,1 \leq i \leq n$, be such that $\sum_{i=1}^{n} a_{i}=1$, where $n \geq 2$. Denote by $w_{j}, 1 \leq j \leq n-1$, the zeros of

$$
f(z)=\sum_{i=1}^{n} \frac{a_{i}}{z-z_{i}}
$$

and let $\Phi: \mathbb{C} \rightarrow \mathbb{R}$ be an arbitrary convex function. Then for any $k \in\{1, \ldots, n-1\}$, $1 \leq m \leq k$, and $\left(t_{1}, \ldots, t_{k}\right) \in \mathbb{C}^{k}$ one has

$$
\begin{aligned}
& \sum_{1 \leq r_{1}<\ldots<r_{k} \leq n-1} \sum_{\pi \in \Sigma_{k}} \Phi\left(\Pi_{k, m}\left(t_{\pi(1)} w_{r_{1}}, \ldots, t_{\pi(k)} w_{r_{k}}\right)\right) \\
\leq & \sum_{1 \leq s_{1}<\ldots<s_{k} \leq n}\left(1-\sum_{i=1}^{k} a_{s_{i}}\right) \sum_{\pi \in \Sigma_{k}} \Phi\left(\Pi_{k, m}\left(t_{\pi(1)} z_{s_{1}}, \ldots, t_{\pi(k)} z_{s_{k}}\right)\right),
\end{aligned}
$$


where $\Pi_{k, m}$ is the $m$-th elementary symmetric function on $k$ symbols. In particular, for any line $L$ in $\mathbb{R}^{3}$ and $\alpha \geq 1$ one has

$$
\begin{aligned}
& \sum_{1 \leq r_{1}<\ldots<r_{k} \leq n-1} I_{\alpha, L}\left(\operatorname{conv}\left(w_{r_{1}}, \ldots, w_{r_{k}}\right)\right) \\
& \leq \sum_{1 \leq s_{1}<\ldots<s_{k} \leq n}\left(1-\sum_{i=1}^{k} a_{s_{i}}\right) I_{\alpha, L}\left(\operatorname{conv}\left(z_{s_{1}}, \ldots, z_{s_{k}}\right)\right) .
\end{aligned}
$$

The fact that (5.2) implies (5.3) follows from (5.1) by taking $m=1$ and integrating over the simplex $\Xi_{k}$. Arguing as in the proof of Corollary 3 one can see that it is actually enough to prove (5.2) for $m=k$ and $k \in\{1, \ldots, n-1\}$. Note also that in the case of equal charges Conjecture 1 would provide a whole new series of inequalities for averages of generalized inertia moments involving the critical points and the zeros of an arbitrary complex polynomial. These would substantially improve both Corollary 5 and the inequalities conjectured in 30, Remark 2.3.9].

Remark 22. In view of the proofs of [26, Theorem 4.7] and Theorem 3 above, a natural way of attacking Conjecture 1 would be to interpret the arguments of $\Phi$ in (5.2) as eigenvalues of some appropriately defined multilinear power " $\wedge^{k} A$ " of a normal matrix $A$ and one of its principal submatrices, where " $\wedge$ " is a suitable multilinear functional on $\otimes^{k} \mathcal{H}$. However, defining such a functional appears to be a nontrivial question. Note for instance that an obvious polarization of the usual antisymmetric tensor power, i.e., setting

$$
A \wedge B=\frac{1}{2}\left[(A+B)^{(2)}-A^{(2)}-B^{(2)}\right]
$$

for square matrices $A, B$ of the same order, cannot lead to the desired conclusion since to begin with one does not get the correct dimensions (these should agree with the number of $k$-tuples in the two sides of (5.2)).

\section{Zeros of Borel Series With positive $l^{1}$-COEFficients}

Theorem 7 applies essentially to any bounded discrete set of positively charged particles with finite total charge for which equilibrium points are known to exist. We conjecture that if the particles accumulate at a point on the boundary of a disk containing all the particles, then there exist infinitely many equilibrium points.

Conjecture 2. Let $\left\{a_{i}\right\}_{i \in \mathbb{N}}$ be a sequence of positive numbers whose sum is finite and $\left\{z_{i}\right\}_{i \in \mathbb{N}}$ a sequence of distinct complex numbers satisfying

$$
\left|z_{i}\right|<\rho \text { for } i \in \mathbb{N} \text { and } \lim _{i \rightarrow \infty} z_{i}=\xi \text { with }|\xi|=\rho
$$

for some fixed positive number $\rho$. Then the function

$$
f(z)=\sum_{i=1}^{\infty} \frac{a_{i}}{z-z_{i}}
$$

has an infinite set of zeros in the disk $\mathbb{D}(\rho)=\{z \in \mathbb{C}:|z|<\rho\}$.

Note that the assumptions of Conjecture 2 imply that condition (3.14) holds and thus $f$ is meromorphic in $\mathbb{D}(\rho)$. Functions representable as a series of the form (6.1) are known as Borel series [33, and so Conjecture 2 may be rephrased as follows: every Borel series with positive $l^{1}$-coefficients whose poles lie in an open disk and accumulate at a point on the boundary of this disk has infinitely many zeros. 
Conjecture 2 may also be translated in operator theoretic terms by using the methods employed in the proofs of Theorems 6 7. Note first that up to a similarity transformation of the complex plane we may assume that $\xi=\rho=1$. Then one can show that the above conjecture is equivalent to the following statement.

Conjecture 3. Let $\left\{z_{n}\right\}_{n \in \mathbb{N}}$ be a sequence of distinct complex numbers in the open unit disk converging to 1 and denote by $A$ the (bounded) normal operator on a separable infinite-dimensional complex Hilbert space $\mathcal{H}$ satisfying $A \mathbf{e}_{n}=z_{n} \mathbf{e}_{n}$, $n \in \mathbb{N}$, where $\left\{\mathbf{e}_{n}\right\}_{n \in \mathbb{N}}$ is an orthonormal basis of $\mathcal{H}$. If $P$ is a bounded operator on $\mathcal{H}$ such that $I-P$ is a rank one orthoprojection, then the compression $\left.P A P\right|_{P \mathcal{H}}$ has infinite point spectrum.

Remark 23. The hypotheses in Conjecture 3 imply that the normal operator $I-A$ is compact, which may facilitate its study. However, it seems likely that Conjecture 2 should actually hold even under the less restrictive conditions $\left|z_{i}\right|<\rho$ for all $i \in \mathbb{N}$ and $\lim _{i \rightarrow \infty}\left|z_{i}\right|=\rho$. In other words, it is reasonable to believe that the convergence condition in Conjecture 3 may be relaxed as follows: $\left|z_{n}\right|<1$ for all $n \in \mathbb{N}$ and $\lim _{n \rightarrow \infty}\left|z_{n}\right|=1$.

\section{Operator versions of the Clunie-Eremenko-Rossi Conjecture}

Various results concerning the existence of equilibrium points for Newtonian and logarithmic potentials have been obtained in, e.g., 8], [14] and [24]. It should be emphasized though that these deal almost exclusively with unbounded discrete charge configurations. Note that Conjecture 2 may actually be viewed as a natural analog for bounded discrete charge configurations of the following well-known conjecture of Clunie-Eremenko-Rossi; see, e.g., [8, Conjecture 2.7] and [24, Conjecture 1.1].

Conjecture 4 (Clunie-Eremenko-Rossi conjecture). Let $\left\{a_{i}\right\}_{i \in \mathbb{N}}$ be a sequence of positive numbers and $\left\{z_{i}\right\}_{i \in \mathbb{N}}$ a sequence of distinct complex numbers such that

$$
z_{i} \rightarrow \infty \text { as } i \rightarrow \infty \text { and } \sum_{z_{i} \neq 0} \frac{a_{i}}{\left|z_{i}\right|}<\infty .
$$

Then the meromorphic function

$$
f(z)=\sum_{i=1}^{\infty} \frac{a_{i}}{z-z_{i}}
$$

has infinitely many zeros.

A catchy albeit somewhat loose rephrasing of this conjecture is as follows: every flat universe has infinitely many resting points.

Let us use the framework of 4.3 to give an operator theoretic interpretation and generalization of Conjecture 4. Note first that up to a translation of the variable $z$ and a rescaling of the coefficients $a_{n}$ we may assume wlog that all $z_{n}$ are nonzero and satisfy the normalization condition

$$
\sum_{i=1}^{\infty} \frac{a_{i}}{\left|z_{i}\right|}=1
$$

As in 4.3 we use the Hilbert space $\mathcal{H}=l^{2}(\mathbb{N})$ with standard inner product $\langle\cdot, \cdot\rangle$ and standard complete orthonormal system $\left\{\mathbf{e}_{n}\right\}_{n \in \mathbb{N}}$. Set $z_{n}=r_{n} e^{i \theta_{n}}, n \in \mathbb{N}$, and 
let $A$ and $B$ be the bounded normal operators on $\mathcal{H}$ satisfying

$$
A \mathbf{e}_{n}=e^{i \theta_{n}} \mathbf{e}_{n}, \quad B_{n}=r_{n}^{-1} \mathbf{e}_{n}, \quad n \in \mathbb{N} .
$$

Clearly, $A$ is unitary and $B$ is compact. Condition (7.1) allows us to define a unit vector $\mathbf{v} \in \mathcal{H}$ by setting

$$
\mathbf{v}=\sum_{n=1}^{\infty} \sqrt{\frac{a_{n}}{\left|z_{n}\right|}} \mathbf{e}_{n}
$$

and one can easily verify that

$$
\left\langle(A-z B)^{-1} \mathbf{v}, \mathbf{v}\right\rangle=-f(z), \quad z \in \mathbb{C} \backslash\left\{z_{n}\right\}_{n \in \mathbb{N}} .
$$

Set $\mathcal{K}=\mathbf{v}^{\perp}$ and let $P$ be the orthoprojection on $\mathcal{K}$. We use the notation $A^{\prime}$ and $B^{\prime}$ for the compressions to $\mathcal{K}$ of $A$ and $B$, respectively, that is,

$$
A^{\prime}=\left.P A P\right|_{\mathcal{K}}, \quad B^{\prime}=\left.P^{B} P\right|_{\mathcal{K}}
$$

By definition, the set of generalized eigenvalues of $A^{\prime}$ with respect to $B^{\prime}$ is

$$
\Sigma_{B^{\prime}}\left(A^{\prime}\right)=\left\{z \in \mathbb{C} \mid N\left(A^{\prime}-z B^{\prime}\right) \neq\{0\}\right\},
$$

where $N(T)$ denotes as usual the null space of an operator $T$. Let $\mathcal{Z}(f)$ be the zero set of $f$.

Lemma 9. In the above notation one has $\mathcal{Z}(f)=\Sigma_{B^{\prime}}\left(A^{\prime}\right)$.

Proof. We make an appropriate adaption of the argument used in the proof of Lemma 7 Using the fact that the $z_{n}$ are distinct one can check that if $0 \neq \mathbf{u} \in \mathcal{H}$, then $\mathbf{u} \in N(A-\zeta B)$ if and only if $\zeta=z_{n}$ and $\mathbf{u}=\mathbf{e}_{n}$ for some $n \in \mathbb{N}$. However

$$
\left\langle\mathbf{e}_{n}, \mathbf{v}\right\rangle=\sqrt{\frac{a_{n}}{\left|z_{n}\right|}} \neq 0,
$$

which shows that $N(A-\zeta B) \cap \mathcal{K}=\{0\}$ for $\zeta \in \mathbb{C}$. Now let $\mathbf{u} \in \mathcal{K}$ be such that $\left(A^{\prime}-\zeta B^{\prime}\right) \mathbf{u}=0$ for some $\zeta \in \mathbb{C}$. From the identity

$$
(A-\zeta B) \mathbf{u}=(I-P)(A-\zeta B) \mathbf{u}+\left(A^{\prime}-\zeta B^{\prime}\right) \mathbf{u}=\langle(A-\zeta B) \mathbf{u}, \mathbf{v}\rangle \mathbf{v}
$$

and $\langle(A-\zeta B) \mathbf{u}, \mathbf{v}\rangle \neq 0$ it follows that $(A-\zeta B)^{-1} \mathbf{v}=\langle(A-\zeta B) \mathbf{u}, \mathbf{v}\rangle^{-1} \mathbf{u}$. By (17.3) one gets

$$
f(\zeta)=-\langle(A-\zeta B) \mathbf{u}, \mathbf{v}\rangle^{-1}\langle\mathbf{u}, \mathbf{v}\rangle=0,
$$

so that $\zeta \in \mathcal{Z}(f)$. Conversely, let $w \in \mathcal{Z}(f)$. Then $w \notin\left\{z_{n}\right\}_{n \in \mathbb{N}}$ and so $A-w B$ is invertible by (7.2). The vector $\mathbf{u}:=(A-w B)^{-1} \mathbf{v}$ is clearly nonzero and one has $\langle\mathbf{u}, \mathbf{v}\rangle=f(w)=0$, hence $\mathbf{u} \in \mathcal{K}$. Thus

$$
\mathbf{v}=(A-w B) \mathbf{u}=\langle(A-w B) \mathbf{u}, \mathbf{v}\rangle \mathbf{v}+\left(A^{\prime}-w B^{\prime}\right) \mathbf{u},
$$

which shows that $\left(A^{\prime}-w B^{\prime}\right) \mathbf{u} \in \mathbf{v}^{\perp} \cap\{\mathbf{v}\}=\{0\}$ and therefore $w \in \Sigma_{B^{\prime}}\left(A^{\prime}\right)$.

Recall that a vector with all nonzero coordinates in the standard basis $\left\{\mathbf{e}_{n}\right\}_{n \in \mathbb{N}}$ of $\mathcal{H}$ is called totally nonzero. The above discussion shows that the Clunie-EremenkoRossi conjecture (Conjecture 4) is in fact equivalent to the following statement.

Conjecture 5. Let $\left\{\alpha_{n}\right\}_{n \in \mathbb{N}}$ and $\left\{\beta_{n}\right\}_{n \in \mathbb{N}}$ be a sequence of unimodular complex numbers and a sequence of positive numbers converging to 0 , respectively. Assume that $\alpha_{n} \beta_{n}^{-1}$ are distinct, and define a unitary operator $A$ and a compact selfadjoint operator $B$ on $\mathcal{H}$ by setting

$$
A \mathbf{e}_{n}=\alpha_{n} \mathbf{e}_{n}, \quad B \mathbf{e}_{n}=\beta_{n} \mathbf{e}_{n}, \quad n \in \mathbb{N} .
$$


Let $\mathbf{v}$ be a totally nonzero unit vector in $\mathcal{H}$, denote by $P$ the orthoprojection on $\mathcal{K}=\mathbf{v}^{\perp}$ and let $A^{\prime}=\left.P A P\right|_{\mathcal{K}}$ and $B^{\prime}=\left.P B P\right|_{\mathcal{K}}$. Then $\left|\Sigma_{B^{\prime}}\left(A^{\prime}\right)\right|=\infty$, i.e., there exist infinitely many $z \in \mathbb{C}$ such that $N\left(A^{\prime}-z B^{\prime}\right) \neq\{0\}$.

If the vector $\mathbf{v}$ in Conjecture 5 is represented in the standard orthonormal basis of $\mathcal{H}$ as $\mathbf{v}=\sum_{n=1}^{\infty} v_{n} \mathbf{e}_{n}$, then Keldysh's value distribution theorem for meromorphic functions implies that Conjecture 5 is true provided that $\sum_{n=1}^{\infty} \beta_{n}^{-1}\left|v_{n}\right|^{2}<\infty$ (see, e.g., 8 and [18, Ch. 5, Theorem 6.2]).

Conjecture 5 suggests the following more general question: let $A$ be an injective compact normal Hilbert space operator and let $B$ and $C$ be finite-rank operators. Is it true that $\left|\Sigma_{I+C}(A+B)\right|=\infty$, that is, are there infinitely many $z \in \mathbb{C}$ such that $N(A+B-z(I+C)) \neq\{0\}$ ? It is not difficult to see that an affirmative answer to this question would actually imply the validity of the operator theoretic version of the Clunie-Eremenko-Rossi conjecture (Conjecture 5). Indeed, in the notation of Conjecture 5 we may write

$$
\begin{aligned}
A^{\prime}-z B^{\prime}=-z A\left\{A^{-1} B-A^{-1}[\right. & P B(I-P)-(I-P) B] \\
& \left.\quad-z^{-1}\left[I-A^{-1}(P A(I-P)+(I-P) A)\right]\right\} .
\end{aligned}
$$

It remains to note that under the assumptions of Conjecture 5 the operator $A^{-1} B$ is injective, compact and normal while both $A^{-1}[P B(I-P)-(I-P) B]$ and $A^{-1}[P A(I-P)+(I-P) A]$ have rank at most two. However, the answer to the question raised above is negative, as one can see from the following example.

Example 1. Let $J$ be the simple dissipative Volterra operator on $L^{2}(0,1)$ defined by

$$
(J f)(x)=2 i \int_{0}^{x} f(t) d t
$$

and set $A=J+J^{*}, B=J-J^{*}$ and $C=0$. Then $A$ is injective compact selfadjoint, $B$ is a one-dimensional operator while the point spectrum of $A+B=2 J$ is empty (cf. [17, p. 187]), so that in this case $A+B-z(I+C)$ is injective for all $z \in \mathbb{C}$.

Let us finally mention that the existence of equilibrium points for some discrete potentials generated by complex charges was established in, e.g., 24]. We propose the following analog of Conjecture 4 for certain potentials of this type.

Conjecture 6. Let $\left\{a_{n}\right\}_{n \in \mathbb{N}}$ and $\left\{z_{n}\right\}_{n \in \mathbb{N}}$ be sequences of complex numbers such that the $z_{n}$ are distinct and satisfy

$$
a_{n} \bar{z}_{n}>0 \text { for } n \in \mathbb{N}, \quad z_{n} \rightarrow \infty \text { as } n \rightarrow \infty, \quad \sum_{n=1}^{\infty}\left|\frac{a_{n}}{z_{n}}\right|<\infty .
$$

Then the meromorphic function

$$
f(z)=\sum_{n=1}^{\infty} \frac{a_{n}}{z-z_{n}}
$$

has infinitely many zeros.

As in the case of Conjectures 2 and 4 , one can reduce the conjecture stated above to a purely operator theoretic question. Indeed, in the notations of Conjecture 6 
let $A$ denote the injective compact normal operator on $\mathcal{H}$ satisfying $A \mathbf{e}_{n}=z_{n}^{-1} \mathbf{e}_{n}$, $n \in \mathbb{N}$. Define a vector

$$
\mathbf{v}=\sum_{n=1}^{\infty} \sqrt{\left|\frac{a_{n}}{z_{n}}\right|} \mathbf{e}_{n} \in \mathcal{H},
$$

let $P$ be the orthoprojection on $\mathbf{v}^{\perp}$ and set $A^{\prime}=\left.P A P\right|_{\mathbf{v}^{\perp}}$. Then

$$
\left\langle\left(A-z^{-1} I\right)^{-1} \mathbf{v}, \mathbf{v}\right\rangle=z f(z), \quad z \in \mathbb{C}^{*} \backslash\left\{z_{n}\right\}_{n \in \mathbb{N}} .
$$

Arguing as in the proof of Lemma 9 one can show that $\zeta^{-1} \in \mathcal{Z}(f)$ for any $\zeta \in$ $\Sigma_{p}\left(A^{\prime}\right) \backslash\{0\}$, where $\mathcal{Z}(f)$ is the zero set of $f$ and $\Sigma_{p}\left(A^{\prime}\right)$ denotes the point spectrum of $A^{\prime}$. For Conjecture 6 it would therefore be enough to show that $\left|\Sigma_{p}\left(A^{\prime}\right)\right|=\infty$.

\section{ACKNOWLEDGEMENTS}

We are grateful to Mihai Putinar, Nikolai Nikolski, Alexandre Eremenko, Donald Sarason and Boris Shapiro for their interest in this work and valuable remarks, as well as to Semen Malamud for making available his inspiring paper [26]. We would also like to thank the anonymous referee for numerous constructive suggestions and for pointing out several useful references.

\section{REFERENCES}

[1] R. Bhatia, Matrix Analysis, Grad. Texts in Math., Vol. 169, Springer-Verlag, New York, 1997. MR1477662 (98i:15003)

[2] J. Borcea, Spectral order and isotonic differential operators of Laguerre-Pólya type, Ark. Mat., to appear. Preprint math.CA/0404336.

[3] J. Borcea, Maximal and linearly inextensible polynomials, Math. Scand., to appear. Preprint math.CV/0309233.

[4] J. Borcea, Equilibrium points of logarithmic potentials induced by positive charge distributions. II. A conjectural Hausdorff geometric symphony, submitted. Preprint (2005).

[5] O. Bratteli, D. W. Robinson, Operator Algebras and Quantum Statistical Mechanics I, Texts Monogr. Phys., Springer-Verlag, New York, 1979. MR0545651 (81a:46070)

[6] N. G. de Bruijn, T. A. Springer, On the zeros of a polynomial and of its derivative II, Indag. Math. 9 (1947), 264-270. MR0021148 (9:30b)

[7] P. Cartier, J. M. G. Fell, P. A. Meyer, Comparaison des mesures portées par un ensemble convexe compact, Bull Soc. Math. France 92 (1964), 435-445. MR0206193 (34:6014)

[8] J. Clunie, A. Eremenko, J. Rossi, On equilibrium points of logarithmic and Newtonian potentials, J. London Math. Soc. (2) 47 (1993), 309-320. MR.1207951 (94c:31001)

[9] G. Dahl, Matrix majorization, Linear Algebra Appl. 288 (1999), 53-73. MR.1670598 (99m:15024)

[10] C. Davis, Eigenvalues of compressions, Bull. Math. Soc. Sci. Math. Phys. RPR 51 (1959), 3-5. MR0125115 (23:A2421)

[11] N. Dunford, J. T. Schwartz, Linear Operators II, Interscience Publ., New York, 1963. MR0188745 (32:6181)

[12] H. Dym, V. Katsnelson, Contributions of Issai Schur to analysis, in: Studies in Memory of Issai Schur, xci-clxxxviii, Progr. Math., Vol. 210, Birkhäuser Boston, 2003. MR1985190 (2004e:46003)

[13] J. Elton, T. P. Hill, On the basic representation theorem for convex domination of measures, J. Math. Anal. Appl. 228 (1998), 449-466. MR.1663581 (99k:28015)

[14] A. Eremenko, J. Langley, J. Rossi, On the zeros of meromorphic functions of the form $f(z)=\sum_{k=1}^{\infty} \frac{a_{k}}{z-z_{k}}$, J. Analyse Math. 62 (1994), 271-286. MR1269209 (95f:30041)

[15] K. Fan, G. Pall, Imbedding conditions for Hermitian and normal matrices, Canad. Math. J. 9 (1957), 298-304. MR0085216 (19:6e)

[16] P. Fischer, J. A. R. Holbrook, Balayage defined by the nonnegative convex functions, Proc. Amer. Math. Soc. 79 (1980), 445-448. MR0567989 (81f:46012) 
[17] I. C. Gohberg, M. G. Krein, Introduction to the Theory of Linear Nonselfadjoint Operators, in: Transl. Math. Monogr., Vol. 18, Amer. Math. Soc., Providence, RI, 1969. MR0246142 (39:7447)

[18] A. A. Goldberg, I. V. Ostrovskii, Distribution of Values of Meromorphic Functions, Nauka, Moscow, 1970. MR0280720 (43:6439)

[19] G. H. Hardy, J. E. Littlewood, G. Pólya, Inequalities, 2nd edition, Cambridge Univ. Press, Cambridge, UK, 1988. MR0944909 (89d:26016)

[20] H. Hochstadt, One dimensional perturbations of compact operators, Proc. Amer. Math. Soc. 37 (1973), 465-467. MR0310681 (46:9779)

[21] T. Kato, Variation of discrete spectra, Commun. Math. Phys. 111 (1987), 501-504. MR0900507 (88g:47045)

[22] T. Kato, Perturbation Theory for Linear Operators, Springer-Verlag, New York, 1966. MR0203473 (34:3324)

[23] O. D. Kellogg, Foundations of Potential Theory, Springer-Verlag, Berlin-New York, 1967. MR0222317 (36:5369)

[24] J. K. Langley, J. Rossi, Critical points of certain discrete potentials, Complex Var. Theory Appl. 49 (2004), 621-637. MR2088052 (2005f:30059)

[25] S. M. Malamud, An analog of the Poincaré separation theorem for normal matrices and the Gauss-Lucas theorem, Funct. Anal. Appl. 37 (2003), 232-235. MR2021139

[26] S. M. Malamud, Inverse spectral problem for normal matrices and the Gauss-Lucas theorem, Trans. Amer. Math. Soc. 357 (2005), 4043-4064. Preprint math.CV/0304158.

[27] A. W. Marshall, I. Olkin, Inequalities: Theory of Majorization and Its Applications, in: Math. Sci. Engrg., Vol. 143, Academic Press, New York, 1979. MR0552278 (81b:00002)

[28] R. Pereira, Differentiators and the geometry of polynomials, J. Math. Anal. Appl. 285 (2003), 336-348. MR2000158 (2004g:15041)

[29] R. R. Phelps, Lectures on Choquet's Theorem, in: Van Nostrand Math. Studies, No. 7, D. Van Nostrand Co., Princeton, NJ, 1966. MR0193470 (33:1690)

[30] Q. I. Rahman, G. Schmeisser, Analytic Theory of Polynomials, in: London Math. Soc. Monogr. (N. S.), Vol. 26, Oxford Univ. Press, New York, 2002. MR.1954841 (2004b:30015)

[31] S. Sherman, On a theorem of Hardy, Littlewood, Pólya, and Blackwell, Proc. Nat. Acad. Sci. USA 37 (1951), 826-831; Errata, ibid. 38 (1952), 382. MR0045787 (13:633g)

[32] J. Sz-Nagy, Verallgemeinerung der Derivierten in der Geometrie der Polynome, Acta Math. Sci. (Szeged) 13 (1950), 169-178. MR0039838(12:606c)

[33] J. Wolff, Sur les séries $\sum \frac{A_{k}}{z-\alpha_{k}}$, C. R. Acad. Sci. Paris 173 (1921), 1056-1057; ibid., 13271328.

Department of Mathematics, Stockholm University, SE-106 91 Stockholm, Sweden

E-mail address: julius@math.su.se 